\title{
Seminormal forms and Gram determinants for cellular algebras
}

\author{
By Andrew Mathas at Sydney \\ With an appendix by Marcos Soriano at Hannover
}

\begin{abstract}
This paper develops an abstract framework for constructing "seminormal forms" for cellular algebras. That is, given a cellular $R$-algebra $A$ which is equipped with a family of JM-elements we give a general technique for constructing orthogonal bases for $A$, and for all of its irreducible representations, when the JM-elements separate $A$. The seminormal forms for $A$ are defined over the field of fractions of $R$. Significantly, we show that the Gram determinant of each irreducible $A$-module is equal to a product of certain structure constants coming from the seminormal basis of $A$. In the non-separated case we use our seminormal forms to give an explicit basis for a block decomposition of $A$.
\end{abstract}

\section{Introduction}

The purpose of this paper is to give an axiomatic way to construct "seminormal forms" and to compute Gram determinants for the irreducible representations of semisimple cellular algebras. By this we mean that, starting from a given cellular basis $\left\{a_{s t}^{\lambda}\right\}$ for a cellular algebra $A$, we give a new cellular basis $\left\{f_{s t}^{\lambda}\right\}$ for the algebra which is orthogonal with respect to a natural bilinear form on the algebra. This construction also gives a "seminormal basis" for each of the cell modules of the algebra. We show that the Gram determinant of the cell modules (the irreducible $A$-modules) can be computed in terms of the structure constants of the new cellular basis of $A$. Combining these results gives a recipe for computing the Gram determinants of the irreducible $A$-modules.

Of course, we cannot carry out this construction for an arbitrary cellular algebra $A$. Rather, we assume that the cellular algebra comes equipped with a family of "JucysMurphy" elements. These are elements of $A$ which act on the cellular basis of $A$ via upper triangular matrices. We will see that, over a field, the existence of such a basis $\left\{f_{s t}^{\lambda}\right\}$ forces $A$ to be (split) semisimple. The cellular algebras which have JM-elements include the group algebras of the symmetric groups, any split semisimple algebra, the Hecke algebras of type $A$, the $q$-Schur algebras, the (degenerate) Ariki-Koike algebras, the cyclotomic $q$-Schur algebras, the Brauer algebras and the BMW algebras. 
At first sight, our construction appears to be useful only in the semisimple case. However, in the last section of this paper we apply these ideas in the non-semisimple case to construct a third cellular basis $\left\{g_{s t}^{\lambda}\right\}$ of $A$. We show that this basis gives an explicit decomposition of $A$ into a direct sum of smaller cellular subalgebras. In general, these subalgebras need not be indecomposable, however, it turns out that these subalgebras are indecomposable in many of the cases we know about. As an application, we give explicit bases for the block decomposition of the group algebras of the symmetric groups, the Hecke algebras of type $A$, the Ariki-Koike algebras with $q \neq 1$, the degenerate Ariki-Koike algebras and the (cyclotomic) $q$-Schur algebras.

There are many other accounts of seminormal forms in the literature; see, for example, [1], [8], [13], [21]. The main difference between this paper and previous work is that, starting from a cellular basis for an algebra we construct seminormal forms for the entire algebra, rather than just the irreducible modules. The main new results that we obtain are explicit formulae for the Gram determinants of the cell modules in the separated case, and a basis for a block decomposition of the algebra in the non-separated case. The seminormal forms that we construct have the advantage that they are automatically defined over the field of fractions of the base ring.

It follows using the Wedderburn theorem that an algebra has a family of separating JM-elements if and only if it is split semisimple (see Example 2.13). As every split semisimple algebra is cellular this suggests that cellular algebras provide the right framework for studying seminormal forms. There is, however, an important caveat: the set of JM-elements for a cellular algebra is not canonical as it depends heavily on the particular choice of cellular basis. Consequently, to study an algebra using the techniques in this paper one has to first find a cellular basis for the algebra and then find an appropriate set of JM-elements. Neither of these tasks is necessarily easy especially as, ideally, we would like the set of JMelements to be compatible with modular reduction.

In the appendix to this paper, Marcos Soriano, gives an alternative matrix theoretic approach to the theory of seminormal forms. Using only the Cayley-Hamilton theorem he shows that if you have a family of operators acting on a module via upper triangular matrices which satisfy an analogous separation condition then you can construct a complete set of pairwise orthogonal idempotents. This shows that, ultimately, the theory of seminormal forms rests on the Cayley-Hamilton theorem. Note that unlike in our treatment, Soriano does not need to assume that the JM-elements commute or that they are *-invariant.

This paper is organized as follows. In the next section we recall Graham and Lehrer's theory of cellular algebras and define JM-elements for cellular algebras. We then show that any cellular algebra with a family of separating JM-elements is necessarily semisimple and, by way of example, show that most of the well-known cellular algebras have JM-elements. The third section of the paper develops the theory of JM-elements in the separated case, culminating with the construction of a seminormal basis for a cellular algebra and the computation of the Gram determinants of the cell modules. In the last section of the paper we use modular reduction to study the non-separated case. Our main result gives a cellular basis for a decomposition of the original cellular algebra into blocks. Finally, in the appendix Marcos Soriano gives his matrix theoretic approach to the theory of seminormal forms. 


\section{Cellular algebras and JM-elements}

We begin by recalling Graham and Lehrer's [6] definition of a cellular algebra. Let $R$ be a commutative ring with 1 and let $A$ be a unital $R$-algebra and let $K$ be the field of fractions of $R$.

2.1. Definition (Graham and Lehrer). A cell datum for $A$ is a triple $(\Lambda, T, C)$ where $\Lambda=(\Lambda,>)$ is a finite poset, $T(\lambda)$ is a finite set for each $\lambda \in \Lambda$, and

$$
C: \coprod_{\lambda \in \Lambda} T(\lambda) \times T(\lambda) \rightarrow A ; \quad(s, t) \mapsto a_{s t}^{\lambda}
$$

is an injective map (of sets) such that:

(a) $\left\{a_{s t}^{\lambda} \mid \lambda \in \Lambda, s, t \in T(\lambda)\right\}$ is an $R$-free basis of $A$.

(b) For any $x \in A$ and $t \in T(\lambda)$ there exist scalars $r_{t v x} \in R$ such that, for any $s \in T(\lambda)$,

$$
a_{s t}^{\lambda} x \equiv \sum_{v \in T(\lambda)} r_{t v x} a_{s v}^{\lambda} \quad\left(\bmod A^{\lambda}\right)
$$

where $A^{\lambda}$ is the $R$-submodule of $A$ with basis $\left\{a_{y z}^{\mu} \mid \mu>\lambda\right.$ and $\left.y, z \in T(\mu)\right\}$.

(c) The $R$-linear map determined by $*: A \rightarrow A ; a_{s t}^{\lambda}=a_{t s}^{\lambda}$, for all $\lambda \in \Lambda$ and $s, t \in T(\lambda)$, is an anti-isomorphism of $A$.

If a cell datum exists for $A$ then we say that $A$ is a cellular algebra.

Henceforth, we fix a cellular algebra $A$ with cell datum $(\Lambda, T, C)$ as above. We will also assume that $T(\lambda)$ is a poset with ordering $\triangleright_{\lambda}$, for each $\lambda \in \Lambda$. For convenience we set $T(\Lambda)=\coprod_{\lambda \in \Lambda} T(\lambda)$. We consider $T(\Lambda)$ as a poset with the ordering $s \triangleright t$ if either (1) $s, t \in T(\lambda)$, for some $\lambda \in \Lambda$, and $s \triangleright_{\lambda} t$, or $(2) s \in T(\lambda), t \in T(\mu)$ and $\lambda>\mu$. We write $s \unrhd t$ if $s=t$ or $s \triangleright t$. If $s \unrhd t$ we say that $s$ dominates $t$.

Note that, by assumption $A$ is a free $R$-module of finite rank $|T(\Lambda)|$.

Let $A_{K}=A \otimes_{R} K$. As $A$ is free as an $R$-module, $A_{K}$ is a cellular algebra with cellular basis $\left\{a_{s t}^{\lambda} \otimes 1_{K} \mid \lambda \in \Lambda\right.$ and $\left.s, t \in T(\lambda)\right\}$. We consider $A$ as a subalgebra of $A_{K}$ and, abusing notation, we also consider $a_{s t}^{\lambda}$ to be elements of $A_{K}$.

We recall some of the general theory of cellular algebras. First, applying the $*$ involution to part (b) of Definition 2.1 we see that if $y \in A$ and $s \in T(\lambda)$ then there exist scalars $r_{s u y}^{\prime} \in R$ such that, for all $t \in T(\lambda)$,

$$
y a_{s t}^{\lambda} \equiv \sum_{u \in T(\lambda)} r_{s u y}^{\prime} a_{u t}^{\lambda} \quad\left(\bmod A^{\lambda}\right)
$$

Consequently, $A^{\lambda}$ is a two-sided ideal of $A$, for any $\lambda \in \Lambda$.

Next, for each $\lambda \in \Lambda$ define the cell module $C(\lambda)$ to be the free $R$-module with basis $\left\{a_{t}^{\lambda} \mid t \in T(\lambda)\right\}$ and with $A$-action given by 


$$
a_{t}^{\lambda} x=\sum_{v \in T(\lambda)} r_{t v x} a_{v}^{\lambda}
$$

where $r_{t v x}$ is the same scalar which appears in Definition 2.1. As $r_{t v x}$ is independent of $s$ this gives a well-defined $A$-module structure on $C(\lambda)$. The map $\langle,\rangle_{\lambda}: C(\lambda) \times C(\lambda) \rightarrow R$ which is determined by

$$
\left\langle a_{t}^{\lambda}, a_{u}^{\lambda}\right\rangle_{\lambda} a_{s}^{\lambda} v \equiv a_{s t}^{\lambda} a_{u v}^{\lambda} \quad\left(\bmod A^{\lambda}\right)
$$

for $s, t, u, v \in T(\lambda)$, defines a symmetric bilinear form on $C(\lambda)$. This form is associative in the sense that $\langle a x, b\rangle_{\lambda}=\left\langle a, b x^{*}\right\rangle_{\lambda}$, for all $a, b \in C(\lambda)$ and all $x \in A$. From the definitions, for any $s \in T(\lambda)$ the cell module $C(\lambda)$ is naturally isomorphic to the $A$-module spanned by $\left\{a_{s t}^{\lambda}+A^{\lambda} \mid t \in T(\lambda)\right\}$. The isomorphism is the obvious one which sends $a_{t}^{\lambda} \mapsto a_{s t}^{\lambda}+A^{\lambda}$, for $t \in T(\lambda)$.

For $\lambda \in \Lambda$ we define $\operatorname{rad} C(\lambda)=\left\{x \in C(\lambda) \mid\langle x, y\rangle_{\lambda}=0\right.$ for all $\left.y \in C(\lambda)\right\}$. As the bilinear form on $C(\lambda)$ is associative it follows that $\operatorname{rad} C(\lambda)$ is an $A$-submodule of $C(\lambda)$. Graham and Lehrer ([6], Theorem 3.4) show that the $A_{K}$-module $D(\lambda)=C(\lambda) / \operatorname{rad} C(\lambda)$ is absolutely irreducible and that $\{D(\lambda) \neq 0 \mid \lambda \in \Lambda\}$ is a complete set of pairwise nonisomorphic irreducible $A_{K}$-modules.

The proofs of all of these results follow easily from Definition 2.1. For the full details see [6], §2-3, or [15], Chapt. 2.

In this paper we are interested only in those cellular algebras which come equipped with the following elements.

2.4. Definition. A family of JM-elements for $A$ is a set $\left\{L_{1}, \ldots, L_{M}\right\}$ of commuting elements of $A$ together with a set of scalars, $\left\{\mathrm{c}_{t}(i) \in R \mid t \in T(\Lambda)\right.$ and $\left.1 \leqq i \leqq M\right\}$, such that for $i=1, \ldots, M$ we have $L_{i}^{*}=L_{i}$ and, for all $\lambda \in \Lambda$ and $s, t \in T(\lambda)$,

$$
a_{s t}^{\lambda} L_{i} \equiv \mathrm{c}_{t}(i) a_{s t}^{\lambda}+\sum_{v \triangleright t} r_{t v} a_{s v}^{\lambda} \quad\left(\bmod A^{\lambda}\right)
$$

for some $r_{t v} \in R$ (which depend on $i$ ). We call $\mathrm{c}_{t}(i)$ the content of $t$ at $i$.

Implicitly, the JM-elements depend on the choice of cellular basis for $A$.

Notice that we also have the following left-hand analogue of the formula in (2.4):

$$
L_{i} a_{s t}^{\lambda} \equiv \mathrm{c}_{s}(i) a_{s t}^{\lambda}+\sum_{u \triangleright s} r_{s u}^{\prime} a_{u t}^{\lambda} \quad\left(\bmod A^{\lambda}\right)
$$

for some $r_{s u}^{\prime} \in R$.

2.6. Let $\mathscr{L}_{K}$ be the subalgebra of $A_{K}$ which is generated by $\left\{L_{1}, \ldots, L_{M}\right\}$. By definition, $\mathscr{L}_{K}$ is a commutative subalgebra of $A_{K}$. It is easy to see that each $t \in T(\Lambda)$ gives rise to a one dimensional representation $K_{t}$ of $\mathscr{L}_{K}$ on which $L_{i}$ acts as multiplication by $\mathrm{c}_{t}(i)$, for $1 \leqq i \leqq M$. In fact, since $\mathscr{L}_{K}$ is a subalgebra of $A_{K}$, and $A_{K}$ has a filtration by cell modules, it follows that $\left\{K_{t} \mid t \in T(\Lambda)\right\}$ is a complete set of irreducible $\mathscr{L}_{K}$-modules.

These observations give a way of detecting when $D(\lambda) \neq 0$ (cf. [6], Prop. 5.9(i)). 
2.7. Proposition. Let $A_{K}$ be a cellular algebra with a family of JM-elements and fix $\lambda \in \Lambda$, and $s \in T(\lambda)$. Suppose that whenever $t \in T(\Lambda)$ and $s \triangleright t$ then $\mathrm{c}_{t}(i) \neq \mathrm{c}_{s}(i)$, for some $i$ with $1 \leqq i \leqq M$. Then $D(\lambda) \neq 0$.

Proof. By Definition 2.4, for any $\mu \in \Lambda$ the $\mathscr{L}_{K}$-module composition factors of $C(\mu)$ are precisely the modules $\left\{K_{t} \mid t \in T(\mu)\right\}$. Observe that if $u, v \in T(\Lambda)$ then $K_{u} \cong K_{v}$ as $\mathscr{L}_{K}$-modules if and only if $\mathrm{c}_{u}(i)=\mathrm{c}_{v}(i)$, for $1 \leqq i \leqq M$. Therefore, our assumptions imply that $K_{s}$ is not an $\mathscr{L}_{K}$-module composition factor of any cell module $C(\mu)$ whenever $\lambda>\mu$. Consequently, $K_{s}$ is not an $\mathscr{L}_{K}$-module composition factor of $D(\mu)$ whenever $\lambda>\mu$. However, by [6], Prop. 3.6, $D(\mu)$ is a composition factor of $C(\lambda)$ only if $\lambda \geqq \mu$. Therefore, $a_{t}^{\lambda} \notin \operatorname{rad} C(\lambda)$ and, consequently, $D(\lambda) \neq 0$ as claimed.

Motivated by Proposition 2.7, we break our study of cellular algebras with JMelements into two cases depending upon whether or not the condition in Proposition 2.7 is satisfied.

2.8. Definition (Separation condition). Suppose that $A$ is a cellular algebra with JM-elements $\left\{L_{1}, \ldots, L_{M}\right\}$. The JM-elements separate $T(\lambda)$ (over $R$ ) if whenever $s, t \in T(\Lambda)$ and $s \triangleright t$ then $\mathrm{c}_{s}(i) \neq \mathrm{c}_{t}(i)$, for some $i$ with $1 \leqq i \leqq M$.

In essence, the separation condition says that the contents $\mathrm{c}_{t}(i)$ distinguish between the elements of $T(\Lambda)$. Using the argument of Proposition 2.7 we see that the separation condition forces $A_{K}$ to be semisimple.

2.9. Corollary. Suppose that $A_{K}$ is a cellular algebra with a family of JM-elements which separate $T(\Lambda)$. Then $A_{K}$ is (split) semisimple.

Proof. By the general theory of cellular algebras [6], Theorem 3.8, $A_{K}$ is (split) semisimple if and only if $C(\lambda)=D(\lambda)$ for all $\lambda \in \Lambda$. By the argument of Proposition 2.7, the separation condition implies that if $s \in T(\lambda)$ then $K_{s}$ does not occur as an $\mathscr{L}_{K}$-module composition factor of $D(\mu)$ for any $\mu>\lambda$. By [6], Prop. 3.6, $D(\mu)$ is a composition factor of $C(\lambda)$ only if $\lambda \geqq \mu$, so the cell module $C(\lambda)=D(\lambda)$ is irreducible. Hence, $A_{K}$ is semisimple as claimed.

In Example 2.13 below we show that every split semisimple algebra is a cellular algebra with a family of JM-elements which separate $T(\Lambda)$.

2.10. Remark. Corollary 2.9 says that if a cellular algebra $A$ has a family of JMelements which separate $T(\Lambda)$ then $A_{K}$ is split semisimple. Conversely, we show in Example 2.13 below that every split semisimple algebra has a family of JM-elements which separate $T(\Lambda)$. However, if $A$ is semisimple and $A$ has a family of JM-elements then it is not true that the $\mathrm{JM}$-elements must separate $A$; the problem is that an algebra can have different families of JM-elements. As described in Example 2.18 below, the Brauer and BMW algebras both have families of JM-elements. Combined with work of Enyang ([5], Examples 7.1 and 10.1) this shows that there exist BMW and Brauer algebras which are semisimple and have JM-elements which do not separate $T(\Lambda)$.

2.11. Remark. Following ideas of Grojnowski [12], (11.9), and (2.6) we can use the algebra $\mathscr{L}_{K}$ to define formal characters of $A_{K}$-modules as follows. Let $\left\{K_{t} \mid t \in L(\Lambda)\right\}$ be a 
complete set of non-isomorphic irreducible $\mathscr{L}_{K}$-modules, where $L(\Lambda) \subseteq T(\Lambda)$. If $M$ is any $A_{K}$-module let $\left[M: K_{t}\right]$ be the decomposition multiplicity of the irreducible $\mathscr{L}_{K}$-module $K_{t}$ in $M$. Define the formal character of $M$ to be

$$
\operatorname{ch} M=\sum_{t \in L(\Lambda)}\left[M: K_{t}\right] e^{t},
$$

which is element of the free $\mathbb{Z}$-module with basis $\left\{e^{t} \mid t \in L(\Lambda)\right\}$. It would be interesting to know to what extent these characters determine the representations of $A$.

We close this introductory section by giving examples of cellular $R$-algebras which have a family of JM-elements. Rather than starting with the simplest example we start with the motivating example of the symmetric group.

2.12. Example (Symmetric groups). The first example of a family of JM-elements was given by Jucys [11] and, independently, by Murphy [17]. (In fact, these elements first appear in the work of Young [23].) Let $A=R \Theta_{n}$ be the group ring of the symmetric group of degree $n$. Define

$$
L_{i}=(1, i)+(2, i)+\cdots+(i-1, i), \quad \text { for } i=2, \ldots, n
$$

Murphy [17] showed that these elements commute and he studied the action of these elements on the seminormal basis of the Specht modules. The seminormal basis of the Specht modules can be extended to a seminormal basis of $R \mathfrak{S}_{n}$, so Murphy's work shows that the group algebra of the symmetric group fits into our general framework. We do not give further details because a better approach to the symmetric groups is given by the special case $q=1$ of Example 2.15 below which concerns the Hecke algebra of type $A$.

2.13. Example (Semisimple algebras). By Corollary 2.9 every cellular algebra over a field which has a family of JM-elements which separate $T(\Lambda)$ is split semisimple. We show the converse is also true. Note that a cellular algebra is semisimple if and only if it is split semisimple, so non-split semisimple algebras do not arise in our setting. In fact, the appendix shows that in the separated case the existence of a family of JM-elements acting on a module forces absolute irreducibilty, so JM-elements never arise in the non-split case.

Suppose that $A_{K}$ is a split semisimple algebra. Then the Wedderburn basis of matrix units in the simple components of $A_{K}$ is a cellular basis of $A_{K}$. We claim that $A_{K}$ has a family of JM-elements. To see this it is enough to consider the case when $A_{K}=\operatorname{Mat}_{n}(K)$ is the algebra of $n \times n$ matrices over $K$. Let $e_{i j}$ be the elementary matrix which has a 1 in row $i$ and column $j$ and zeros elsewhere. Then it is easy to check that $\left\{e_{i j}\right\}$ is a cellular basis for $A_{K}$ (with $\Lambda=\{1\}$, say, and $T(\lambda)=\{1, \ldots, n\}$ ). Let $L_{i}=e_{i i}$, for $1 \leqq i \leqq n$. Then $\left\{L_{1}, \ldots, L_{n}\right\}$ is a family of JM-elements for $A_{K}$ which separate $T(\Lambda)$.

By the last paragraph, any split semisimple algebra $A_{K}$ has a family of JM-elements $\left\{L_{1}, \ldots, L_{M}\right\}$ which separate $T(\Lambda)$, where $M=d_{1}+\cdots+d_{r}$ and $d_{1}, \ldots, d_{r}$ are the dimensions of the irreducible $A_{K}$-modules. The examples below show that we can often find a much smaller set of JM-elements. In particular, this shows that the number $M$ of JMelements for an algebra is not an invariant of $A$ ! Nevertheless, in the separated case we will show that the JM-elements are always linear combinations of the diagonal elementary 
matrices coming from the different Wedderburn components of the algebra. Further, the subalgebra of $A_{K}$ generated by a family of JM-elements is a maximal abelian subalgebra of $A_{K}$.

If $A_{K}$ is a cellular algebra and explicit formulae for the Wedderburn basis of $A_{K}$ are known then we do not need this paper to understand the representations of $A_{K}$. One of the points of this paper is that if we have a cellular basis for an R-algebra A together with a family of JM-elements then we can construct a Wedderburn basis for $A_{K}$.

2.14. Example (A toy example). Let $A=R[X] /\left(X-c_{1}\right) \ldots\left(X-c_{n}\right)$, where $X$ is an indeterminate over $R$ and $c_{1}, \ldots, c_{n} \in R$. Let $x$ be the image of $X$ in $A$ under the canonical projection $R[X] \rightarrow A$. Set $a_{i}:=a_{i i}^{i}=\prod_{j=1}^{i-1}\left(x-c_{j}\right)$, for $i=1, \ldots, n+1$. Then $A$ is a cellular algebra with $\Lambda=\{1, \ldots, n\}, T(i)=\{i\}$, for $1 \leqq i \leqq n$, and with cellular basis $\left\{a_{11}^{1}, \ldots, a_{n n}^{n}\right\}$. Further, $x$ is a JM-element for $A$ because

$$
a_{i} x=\left(x-c_{1}\right) \ldots\left(x-c_{i-1}\right) x=c_{i} a_{i}+a_{i+1},
$$

for $i=1, \ldots, n$. Thus, $c_{i}(x)=c_{i}$, for all $i$. The 'family' of JM-elements $\{x\}$ separates $T(\Lambda)$ if and only if $c_{1}, \ldots, c_{n}$ are pairwise distinct.

2.15. Example (Hecke algebras of type $A$ ). Fix an integer $n>1$ and an invertible element $q \in R$. Let $\mathscr{H}=\mathscr{H}_{R, q}\left(\Xi_{n}\right)$ be the Hecke algebra of type $A$. In particular, if $q=1$ then $\mathscr{H}_{R, q}\left(\Xi_{n}\right) \cong R \Im_{n}$. In general, $\mathscr{H}$ is free as an $R$-module with basis $\left\{T_{w} \mid w \in \Xi_{n}\right\}$ and with multiplication determined by

$$
T_{(i, i+1)} T_{w}= \begin{cases}T_{(i, i+1) w}, & \text { if } i^{w}>(i+1)^{w} \\ q T_{(i, i+1) w}+(q-1) T_{w}, & \text { otherwise }\end{cases}
$$

Recall that a partition of $n$ is a weakly decreasing sequence of positive integers which sum to $n$. Let $\Lambda$ be the set of partitions of $n$ ordered by dominance ([15], 3.5). If $\lambda=\left(\lambda_{1}, \ldots, \lambda_{k}\right)$ is a partition let $[\lambda]=\left\{(r, c) \mid 1 \leqq c \leqq \lambda_{r}, r \leqq k\right\}$ be the diagram of $\lambda$. A standard $\lambda$-tableau is a map $t:[\lambda] \rightarrow\{1, \ldots, n\}$ such that $t$ is monotonic increasing in both coordinates (i.e. rows and columns).

Given $\lambda \in \Lambda$ let $T(\lambda)$ be the set of standard $\lambda$-tableau, ordered by dominance (the Bruhat order; see [15], Theorem 3.8). Murphy [19] has shown that $\mathscr{H}$ has a cellular basis of the form $\left\{m_{s t}^{\lambda} \mid \lambda \in \Lambda\right.$ and $\left.s, t \in T(\lambda)\right\}$.

Set $L_{1}=0$ and define

$$
L_{i}=\sum_{j=1}^{i-1} q^{j-i} T_{(i, j)}, \quad \text { for } 2 \leqq i \leqq n
$$

It is a straightforward, albeit tedious, exercise to check that these elements commute; see, for example, [15], Prop. 3.26. The cellular algebra $*$ involution of $\mathscr{H}$ is the linear extension of the map which sends $T_{w}$ to $T_{w^{-1}}$, for $w \in \Theta_{n}$. So $L_{i}^{*}=L_{i}$, for all $i$. 
For any integer $k$ let $[k]_{q}=1+q+\cdots+q^{k-1}$ if $k \geqq 0$ and set $[k]_{q}=-q^{-k}[-k]_{q}$ if $k<0$. Let $t$ be a standard tableau and suppose that $i$ appears in row $r$ and column $c$ of $t$, where $1 \leqq i \leqq n$. The $q$-content of $i$ in $t$ is $\mathrm{c}_{t}(i)=[c-r]_{q}$. Then, by [15], Theorem 3.32,

$$
m_{s t}^{\lambda} L_{i}=\mathrm{c}_{t}(i) m_{s t}^{\lambda}+\text { more dominant terms. }
$$

Hence, $\left\{L_{1}, \ldots, L_{n}\right\}$ is a family of JM-elements for $\mathscr{H}$. Moreover, if $[1]_{q}[2]_{q} \ldots[n]_{q} \neq 0$ then a straightforward induction shows that the JM-elements separate $T(\Lambda)$; see [15], Lemma 3.34.

2.16. Example (Ariki-Koike algebras). Fix integers $n, m \geqq 1$, an invertible element $q \in R$ and an $m$-tuple $\boldsymbol{u}=\left(u_{1}, \ldots, u_{m}\right) \in R^{m}$. The Ariki-Koike algebra $\mathscr{H}_{R, q, \boldsymbol{u}}$ is a deformation of the group algebra of the complex reflection group of type $G(m, 1, n)$; that is, the group $(\mathbb{Z} / m \mathbb{Z})$ \& $\Xi_{n}$. The Ariki-Koike algebras are generated by elements $T_{0}, T_{1}, \ldots, T_{n-1}$ subject to the relations $\left(T_{0}-u_{1}\right) \ldots\left(T_{0}-u_{m}\right)=0,\left(T_{i}-q\right)\left(T_{i}+1\right)=0$ for $1 \leqq i<n$, together with the braid relations of type $B$.

Let $\Lambda$ be the set of m-multipartitions of $n$; that is, the set of $m$-tuples of partitions which sum to $n$. Then $\Lambda$ is a poset ordered by dominance. If $\lambda \in \Lambda$ then a standard $\lambda$-tableau is an $m$-tuple of standard tableau $t=\left(t^{(1)}, \ldots, t^{(m)}\right)$ which, collectively, contain the numbers $1, \ldots, n$ and where $t^{(s)}$ has shape $\lambda^{(s)}$. Let $T(\lambda)$ be the set of standard $\lambda$-tableaux ordered by dominance $([4],(3.11))$. It is shown in [4] that the Ariki-Koike algebra has a cellular basis of the form $\left\{m_{s t}^{\lambda} \mid \lambda \in \Lambda\right.$ and $\left.s, t \in T(\lambda)\right\}$.

For $i=1, \ldots, n$ set $L_{i}=q^{1-i} T_{i-1} \ldots T_{1} T_{i} T_{1} \ldots T_{i-1}$. These elements commute, are invariant under the $*$ involution of $\mathscr{H}_{R, q, \boldsymbol{u}}$ and

$$
m_{s t}^{\lambda} L_{i}=\mathrm{c}_{t}(i) m_{s t}^{\lambda}+\text { more dominant terms, }
$$

where $\mathrm{c}_{t}(i)=u_{s} q^{c-r}$ if $i$ appears in row $r$ and column $c$ of $t^{(s)}$. All of these facts are proved in [10], §3. Hence, $\left\{L_{1}, \ldots, L_{n}\right\}$ is a family of JM-elements for $\mathscr{H}_{R, q, \boldsymbol{u}}$. In this case, if $[1]_{q} \ldots[n]_{q} \prod_{1 \leqq i<j \leqq m|d|<n} \prod_{\mid d}\left(q^{d} u_{i}-u_{j}\right) \neq 0$ and $q \neq 1$ then the JM-elements separate $T(\Lambda)$ by [10], Lemma 3.12.

There is an analogous family of JM-elements for the degenerate Ariki-Koike algebras. See [2], §6, for details.

2.17. Example (Schur algebras). Let $\Lambda$ be the set of partitions of $n$, ordered by dominance, and for $\mu \in \Lambda$ let $\mathfrak{\Xi}_{\mu}$ be the corresponding Young subgroup of $\mathfrak{\Xi}_{n}$ and set $m_{\mu}=\sum_{w \in \mathfrak{\subseteq}_{\mu}} T_{w} \in \mathscr{H}$. Then the $q$-Schur algebra is the endomorphism algebra

$$
\mathrm{S}_{R, q}(n)=\operatorname{End}_{\mathscr{H}}\left(\bigoplus_{\mu \in \Lambda} m_{\mu} \mathscr{H}\right) .
$$

For $\lambda \in \Lambda$ let $T(\lambda)$ be the set of semistandard $\lambda$-tableaux, and let $T_{\mu}(\lambda) \subseteq T(\lambda)$ be the set of semistandard $\lambda$-tableaux of type $\mu$; see [15], $\$ 4.1$. The main result of [4] says that $\mathrm{S}_{R, q}(n)$ has a cellular basis $\left\{\varphi_{S T}^{\lambda} \mid \lambda \in \Lambda\right.$ and $\left.S, T \in T(\lambda)\right\}$ where the homomorphism $\varphi_{S T}^{\lambda}$ is given by left multiplication by a sum of Murphy basis elements $m_{s t}^{\lambda} \in \mathscr{H}$ which depend on $S$ and $T$. 
Let $\mu=\left(\mu_{1}, \ldots, \mu_{k}\right)$ be a partition in $\Lambda$. For $i=1, \ldots, k$ let $L_{i}^{\mu}$ be the endomorphism of $m_{\mu} \mathscr{H}$ which is given by

$$
L_{i}^{\mu}\left(m_{\mu} h\right)=\sum_{j=\mu_{1}+\cdots+\mu_{i-1}+1}^{\mu_{1}+\cdots+\mu_{i}} L_{j} m_{\mu} h
$$

for all $h \in \mathscr{H}$. Here, $L_{1}, \ldots, L_{n}$ are the JM-elements of the Hecke algebra $\mathscr{H}$. We can consider $L_{i}^{\mu}$ to be an element of $S_{R, q}(n)$. Using properties of the JM-elements of $\mathscr{H}$ it is easy to check that the $L_{i}^{\mu}$ commute, that they are $*$-invariant and by [9], Theorem 3.16 , that

$$
\varphi_{S T}^{\lambda} L_{i}^{\mu}= \begin{cases}\mathrm{c}_{T}(i) \varphi_{S T}^{\lambda}+\text { more dominant terms, } & \text { if } T \in T_{\mu}(\lambda) \\ 0, & \text { otherwise }\end{cases}
$$

Here $\mathrm{c}_{T}(i)$ is the sum of the $q$-contents of the nodes in $T$ labelled by $i$ ([15], §5.1). Hence $\left\{L_{i}^{\mu} \mid \mu \in \Lambda\right\}$ is a family of JM-elements for $\mathrm{S}_{R, q}(n)$. If $[1]_{q} \ldots[n]_{q} \neq 0$ then the JM-elements separate $T(\Lambda)$; see [15], Lemma 5.4.

More generally, the $q$-Schur algebras $S_{R, q}(n, r)$ of type $A$ and the cyclotomic $q$-Schur algebras both have a family of JM-elements; see [9], [10] for details.

2.18. Example (Birman-Murakami-Wenzl algebras). Let $r$ and $q$ be invertible indeterminates over $R$ and let $n \geqq 1$ an integer. Let $\mathscr{B}_{n}(q, r)$ be the Birman-Murakami-Wenzl algebra, or BMW algebra. The BMW algebra is generated by elements $T_{1}, \ldots, T_{n-1}$ which satisfy the relations $\left(T_{i}-q\right)\left(T_{i}+q^{-1}\right)\left(T_{i}-r^{-1}\right)=0$, the braid relations of type $A$, and the relations $E_{i} T_{i \pm 1}^{ \pm 1} E_{i}=r^{ \pm 1} E_{i}$ and $E_{i} T_{i}=T_{i} E_{i}=r^{-1} E_{i}$, where $E_{i}=1-\frac{T_{i}-T_{i}^{-1}}{q-q^{-1}}$; see [5],
[13].

The BMW algebra $\mathscr{B}_{n}(q, r)$ is a deformation of the Brauer algebra. Indeed, both the Brauer and BMW algebras have a natural diagram basis indexed by the set of $n$-Brauer diagrams; that is, graphs with vertex set $\{1, \ldots, n, \overline{1}, \ldots, \bar{n}\}$ such that each vertex lies on a unique edge. For more details see [7].

Let $\lambda$ be a partition of $n-2 k$, where $0 \leqq k \leqq\left\lfloor\frac{n}{2}\right\rfloor$. An $n$-updown $\lambda$-tableau $t$ is an $n$-tuple $t=\left(t_{1}, \ldots, t_{n}\right)$ of partitions such that $t_{1}=(1), t_{n}=\lambda$ and $\left|t_{i}\right|=\left|t_{i-1}\right| \pm 1$, for $2 \leqq i \leqq n$. (Here $\left|t_{i}\right|$ is the sum of the parts of the partition $t_{i}$.)

Let $\Lambda$ be the set of partitions of $n-2 k$, for $0 \leqq k \leqq\left\lfloor\frac{n}{2}\right\rfloor$ ordered again by dominance. For $\lambda \in \Lambda$ let $T(\lambda)$ be the set of $n$-updown tableaux. Enyang ([5], Theorem 4.8 and $\S 5$ ) has given an algorithm for constructing a cellular basis of $\mathscr{B}_{n}(q, r)$ of the form $\left\{m_{s t}^{\lambda} \mid \lambda \in \Lambda\right.$ and $\left.s, t \in T(\lambda)\right\}$. Enyang actually constructs a basis for each cell module of $\mathscr{B}_{n}(q, r)$ which is "compatible" with restriction, however, his arguments give a new cellular basis $\left\{m_{s t}^{\lambda}\right\}$ for $\mathscr{B}_{n}(q, r)$ which is indexed by pairs of $n$-updown $\lambda$-tableaux for $\lambda \in \Lambda$.

Following [13], Cor. 1.6, set $L_{1}=1$ and define $L_{i+1}=T_{i} L_{i} T_{i}$, for $i=2, \ldots, n$. These elements are invariant under the $*$ involution of $\mathscr{B}_{n}(q, r)$ and Enyang $([5], \S 6)$ has shown 
that $L_{1}, \ldots, L_{n}$ commute and that

$$
m_{s t}^{\lambda} L_{i}=\mathrm{c}_{t}(i) m_{s t}^{\lambda}+\text { more dominant terms, }
$$

where $\mathrm{c}_{t}(i)=q^{2(c-b)}$ if $\left[t_{i}\right]=\left[t_{i-1}\right] \cup\{(b, c)\}$ and $\mathrm{c}_{t}(i)=r^{-2} q^{2(b-c)}$ if $\left[t_{i}\right]=\left[t_{i-1}\right] \backslash\{(b, c)\}$. Hence, $L_{1}, \ldots, L_{n}$ is a family of JM-elements for $\mathscr{B}_{n}(q, r)$. When $R=\mathbb{Z}\left[r^{ \pm 1}, q^{ \pm 1}\right]$ the JMelements separate $T(\Lambda)$.

The BMW algebras include the Brauer algebras essentially as a special case. Indeed, it follows from Enyang's work [5], §8-9, that the Brauer algebras have a family of JMelements which separate $T(\Lambda)$.

Rui and Si [22] have recently computed the Gram determinants of the irreducible modules of the Brauer algebras in the semisimple case.

It should be possible to find JM-elements for other cellular algebras such as the partition algebras and the cyclotomic Nazarov-Wenzl algebras [2].

\section{The separated case}

Throughout this section we assume that $A$ is a cellular algebra with a family of JMelements which separate $T(\Lambda)$ over $R$. By Corollary 2.9 this implies that $A_{K}$ is a split semisimple algebra.

For $i=1, \ldots, M$ let $\mathscr{C}(i)=\left\{\mathrm{c}_{t}(i) \mid t \in T(\Lambda)\right\}$. Thus, $\mathscr{C}(i)$ is the set of possible contents that the elements of $T(\Lambda)$ can take at $i$.

We can now make the key definition of this paper.

3.1. Definition. Suppose that $s, t \in T(\lambda)$, for some $\lambda \in \Lambda$ and define

$$
F_{t}=\prod_{i=1}^{M} \prod_{\substack{c \in \mathscr{C}(i) \\ c \neq c_{t}(i)}} \frac{L_{i}-c}{c_{t}(i)-c} .
$$

Thus, $F_{t} \in A_{K}$. Define $f_{s t}^{\lambda}=F_{s} a_{s t}^{\lambda} F_{t} \in A_{K}$.

3.2. Remark. Rather than working over $K$ we could instead work over a ring $R^{\prime}$ in which the elements $\left\{\mathrm{c}_{s}(i)-\mathrm{c}_{t}(i) \mid s \neq t \in T(\Lambda)\right.$ and $\left.1 \leqq i \leqq M\right\}$ are invertible. All of the results below, except those concerned with the irreducibe $A_{K}$-modules or with the semisimplicity of $A_{K}$, are valid over $R^{\prime}$. However, there seems to be no real advantage to working over $R^{\prime}$ in this section. In section 4 we work over a similar ring when studying the nonseparated case. We extend the dominance order $\triangleright$ on $T(\Lambda)$ to $\coprod_{\lambda \in \Lambda} T(\lambda) \times T(\lambda)$ by declaring that
$(s, t) \triangleright(u, v)$ if $s \unrhd u, t \unrhd v$ and $(s, t) \neq(u, v)$.

We now begin to apply our definitions. The first step is easy.

3.3. Lemma. Assume that $A$ has a family of JM-elements which separate $T(\Lambda)$. 
(a) Suppose that $s, t \in T(\lambda)$. Then there exist scalars $b_{w v} \in K$ such that

$$
f_{s t}^{\lambda}=a_{s t}^{\lambda}+\sum_{\substack{u, v \in T(\mu), \mu \in \Lambda \\(u, v) \triangleright(s, t)}} b_{u v} a_{u v}^{\mu} .
$$

(b) $\left\{f_{s t}^{\lambda} \mid s, t \in T(\lambda)\right.$ for some $\left.\lambda \in \Lambda\right\}$ is a basis of $A_{K}$.

(c) Suppose that $s, t \in T(\lambda)$. Then $\left(f_{s t}^{\lambda}\right)^{*}=f_{t s}^{\lambda}$.

Proof. By the definition of the JM-elements (2.4), for any $i$ and any $c \in \mathscr{C}(i)$ with $c \neq \mathrm{c}_{t}(i)$ we have

$$
a_{s t}^{\lambda} \frac{L_{i}-c}{c_{t}(i)-c} \equiv a_{s t}^{\lambda}+\sum_{v \triangleright t} b_{v} a_{s v}^{\lambda} \quad\left(\bmod A_{K}^{\lambda}\right)
$$

By (2.5) this is still true if we act on $a_{s t}^{\lambda}$ with $L_{i}$ from the left. These two facts imply part (a). Note that part (a) says that the transition matrix between the two bases $\left\{a_{s t}^{\lambda}\right\}$ and $\left\{f_{s t}^{\lambda}\right\}$ of $A_{K}$ is unitriangular (when the rows and columns are suitably ordered). Hence, (b) follows. Part (c) follows because, by definition, $\left(a_{s t}^{\lambda}\right)^{*}=a_{t s}^{\lambda}$ and $L_{i}^{*}=L_{i}$, so that $F_{t}^{*}=F_{t}$ and $\left(f_{s t}^{\lambda}\right)^{*}=F_{t} a_{t s}^{\lambda} F_{s}=f_{t s}^{\lambda}$.

Given $s, t \in T(\Lambda)$ let $\delta_{s t}$ be the Kronecker delta; that is, $\delta_{s t}=1$ if $s=t$ and $\delta_{s t}=0$, otherwise.

3.4. Proposition. Suppose that $s, t \in T(\lambda)$, for some $\lambda \in \Lambda$, that $u \in T(\Lambda)$ and fix $i$ with $1 \leqq i \leqq M$. Then
(a) $f_{s t}^{\lambda} L_{i}=c_{t}(i) f_{s t}^{\lambda}$,
(c) $L_{i} f_{s t}^{\lambda}=c_{s}(i) f_{s t}^{\lambda}$,
(b) $f_{s t}^{\lambda} F_{u}=\delta_{t u} f_{s u}^{\lambda}$
(d) $F_{u} f_{s t}^{\lambda}=\delta_{u s} f_{u t}^{\lambda}$.

Proof. Notice that statements (a) and (c) are equivalent by applying the $*$ involution. Similarly, (b) and (d) are equivalent. Thus, it is enough to show that (a) and (b) hold. Rather than proving this directly we take a slight detour.

Let $N=|T(\Lambda)|$ and fix $v=v_{1} \in T(\mu)$ with $v \triangleright t$. We claim that $a_{u v}^{\mu} F_{t}^{N}=0$, for all $u \in T(\mu)$. By the separation condition (2.8), there exists an integer $j_{1}$ with $\mathrm{c}_{t}\left(j_{1}\right) \neq \mathrm{c}_{v}\left(j_{1}\right)$. Therefore, by 2.4, $a_{u v}^{\mu}\left(L_{j_{1}}-\mathrm{c}_{v}\left(j_{1}\right)\right)$ is a linear combination of terms $a_{w x}^{v}$, where $x \triangleright v \triangleright t$. However, $\left(L_{j_{1}}-c_{v}\left(j_{1}\right)\right)$ is a factor of $F_{t}$, so $a_{u v}^{\mu} F_{t}$ is a linear combination of terms of the form $a_{w x}^{v}$ where $x \triangleright v \triangleright t$. Let $v_{2} \in T\left(\mu_{2}\right)$ be minimal such that $a_{u_{2} v_{2}}^{\mu_{2}}$ appears with nonzero coefficient in $a_{u v}^{\mu} F_{t}$, for some $u_{2} \in T\left(\mu_{2}\right)$. Then $v_{2} \triangleright v_{1} \triangleright t$, so there exists an integer $j_{2}$ such that $c_{t}\left(j_{2}\right) \neq c_{v_{2}}\left(j_{2}\right)$. Consequently, $\left(L_{j_{2}}-c_{v_{2}}\left(j_{2}\right)\right)$ is a factor of $F_{t}$, so $a_{u v}^{\mu} F_{t}^{2}$ is a linear combination of terms of the form $a_{w x}^{v}$, where $x \triangleright v_{2} \triangleright v_{1} \triangleright t$. Continuing in this way proves the claim.

For any $s, t \in T(\lambda)$ let $f_{s t}^{\prime}=F_{s}^{N} a_{s t}^{\lambda} F_{t}^{N}$. Fix $j$ with $1 \leqq j \leqq M$. Then, because the JMelements commute,

$$
f_{s t}^{\prime} L_{j}=F_{s}^{N} a_{s t}^{\lambda} F_{t}^{N} L_{j}=F_{s}^{N} a_{s t}^{\lambda} L_{j} F_{t}^{N}=F_{s}^{N}\left(c_{t}(i) a_{s t}^{\lambda}+x\right) F_{t}^{N},
$$


where $x$ is a linear combination of terms of the form $a_{u v}^{\mu}$ with $v \triangleright t$ and $u, v \in T(\mu)$ for some $\mu \in \Lambda$. However, by the last paragraph $x F_{t}^{N}=0$, so this implies that $f_{s t}^{\prime} L_{j}=\mathrm{c}_{t}(j) f_{s t}^{\prime}$. Consequently, every factor of $F_{t}$ fixes $f_{s t}^{\prime}$, so $f_{s t}^{\prime}=f_{s t}^{\prime} F_{t}$. Moreover, if $u \neq t$ then we can find $j$ such that $\mathrm{c}_{t}(j) \neq \mathrm{c}_{u}(j)$ by the separation condition, so that $f_{s t}^{\prime} F_{u}=0$ since $\left(L_{j}-c_{u}(j)\right)$ is a factor of $F_{u}$. As $F_{u} f_{s t}^{\prime}=\left(f_{t s}^{\prime} F_{u}\right)^{*}$, we have shown that

$$
F_{u} f_{s t}^{\prime} F_{v}=\delta_{u s} \delta_{t v} f_{s t}^{\prime}
$$

for any $u, v \in T(\Lambda)$.

We are now almost done. By the argument of Lemma 3.3(a) we know that

$$
f_{s t}^{\prime}=a_{s t}^{\lambda}+\sum_{\substack{u, v \in T(\mu) \\(u, v) \triangleright(s, t)}} s_{u v} a_{u v}^{\mu}
$$

for some $s_{u v} \in K$. Inverting this equation we can write

$$
a_{s t}^{\lambda}=f_{s t}^{\prime}+\sum_{\substack{u, v \in T(\mu) \\(u, v) \triangleright(s, t)}} s_{u v}^{\prime} f_{u v}^{\prime}
$$

for some $s_{u v}^{\prime} \in K$. Therefore,

$$
f_{s t}^{\lambda}=F_{s} a_{s t}^{\lambda} F_{t}=F_{s}\left(f_{s t}^{\prime}+\sum_{\substack{u, v \in T(\mu) \\(u, v) \triangleright(s, t)}} s_{u v}^{\prime} f_{u v}^{\prime}\right) F_{t}=F_{s} f_{s t}^{\prime} F_{t}=f_{s t}^{\prime},
$$

where the last two equalities follow from (3.5). That is, $f_{s t}^{\lambda}=f_{s t}^{\prime}$. We now have that

$$
f_{s t}^{\lambda} L_{i}=f_{s t}^{\prime} L_{i}=\mathrm{c}_{t}(i) f_{s t}^{\prime}=\mathrm{c}_{t}(i) f_{s t}^{\lambda}
$$

proving (a). Finally, if $u \in T(\Lambda)$ then

$$
f_{s t}^{\lambda} F_{u}=f_{s t}^{\prime} F_{u}=\delta_{t u} f_{s t}^{\prime}=\delta_{t u} f_{s t}^{\lambda}
$$

proving (b). (In fact, (b) also follows from (a) and the separation condition.)

3.6. Remark. The proof of Proposition 3.4 is the only place where we explicitly invoke the separation condition. All of the results which follow rely on this key result. It is worth noting the proof of Proposition 3.4 relies on the assumptions that the $L_{1}, \ldots, L_{M}$ commute and that $L_{i}^{*}=L_{i}$, for $\leqq i \leqq M$. The commutativity of the JM-elements is essential in proving 3.4. If we did not assume that $L_{i} \neq L_{i}^{*}$ then we could define $f_{s t}=F_{s}^{*} a_{s t}^{\lambda} F_{t}$. If we did this then in order to prove that $f_{s t}^{\prime} F_{u}=0$ we would have to assume that the $L_{i}^{*}$ act from the right on the basis $\left\{a_{v}^{\mu}\right\}$ in essentially the same way as the $L_{j}$ do. We note that neither of these assumptions appear in Soriano's treatment in the appendix.

3.7. Theorem. Suppose that the JM-elements separate $T(\Lambda)$ over $R$. Let $s, t \in T(\lambda)$ and $u, v \in T(\mu)$, for some $\lambda, \mu \in \Lambda$. Then there exist scalars $\left\{\gamma_{t} \in K \mid t \in T(\Lambda)\right\}$ such that

$$
f_{s t}^{\lambda} f_{u v}^{\mu}= \begin{cases}\gamma_{t} f_{s v}^{\lambda}, & \text { if } \lambda=\mu \text { and } t=u, \\ 0, & \text { otherwise. }\end{cases}
$$


In particular, $\gamma_{t}$ depends only on $t \in T(\Lambda)$ and $\left\{f_{s t}^{\lambda} \mid s, t \in T(\lambda)\right.$ and $\left.\lambda \in \Lambda\right\}$ is a cellular basis of $A_{K}$.

Proof. Using the definitions, $f_{s t}^{\lambda} f_{u v}^{\mu}=f_{s t}^{\lambda} F_{u} a_{u v}^{\mu} F_{v}$. So $f_{s t}^{\lambda} f_{u v}^{\mu} \neq 0$ only if $u=t$ by Proposition $3.4(\mathrm{~b})$.

Now suppose that $u=t$ (so that $\mu=\lambda$ ). Using Lemma 3.3, we can write $f_{s t}^{\lambda} f_{t v}^{\lambda}=\sum_{w, x} r_{w x} f_{w x}^{\mu}$, where $r_{w x} \in R$ and the sum is over pairs $w, x \in T(\mu)$, for some $\mu \in \Lambda$. Hence, by parts (b) and (d) of Proposition 3.4

$$
f_{s t}^{\lambda} f_{t v}^{\lambda}=F_{s} f_{s t}^{\lambda} f_{t v}^{\lambda} F_{v}=\sum_{\substack{\mu \in \Lambda \\ w, x \in T(\mu)}} r_{w x} F_{S} f_{w x}^{\mu} F_{v}=r_{s v} f_{s v}^{\lambda} .
$$

Thus, it remains to show that scalar $r_{s v}$ is independent of $s, v \in T(\lambda)$. Using Lemma 3.3 to compute directly, there exist scalars $b_{w x}, c_{y z}, d_{w x} \in K$ such that

$$
\begin{aligned}
f_{s t}^{\lambda} f_{t v}^{\lambda} \equiv & \left(a_{s t}^{\lambda}+\sum_{\substack{w, x \in T(\lambda) \\
(w, x) \triangleright(s, t)}} b_{w x} a_{w x}^{\lambda}\right)\left(a_{t v}^{\lambda}+\sum_{\substack{y, z \in T(\lambda) \\
y, z) \triangleright(t, v)}} c_{y z} a_{y z}^{\lambda}\right) \bmod A_{K}^{\lambda} \\
\equiv & \left(\left\langle a_{t}^{\lambda}, a_{t}^{\lambda}\right\rangle_{\lambda}+\sum_{\substack{u \in T(\lambda) \\
u \triangleright t}}\left(b_{s u}+c_{u v}\right)\left\langle a_{u}^{\lambda}, a_{t}^{\lambda}\right\rangle_{\lambda}+\sum_{\substack{x, y \in T(\lambda) \\
x, y \triangleright t}} b_{s x} c_{y v}\left\langle a_{x}^{\lambda}, a_{y}^{\lambda}\right\rangle_{\lambda}\right) a_{s v}^{\lambda} \\
& +\sum_{\substack{w, x \in T(\lambda) \\
(w, x) \triangleright(s, v)}} d_{w x} a_{w x}^{\lambda}\left(\bmod A_{K}^{\lambda}\right) .
\end{aligned}
$$

The inner products in the last equation come from applying (2.3). (For typographical convenience we also use the fact that the form is symmetric in the sum over $u$.) By the cellular basis axiom 2.1(b) the scalars $b_{s u}$ and $c_{u v}$ do not depend on $s$ or $v$. Therefore, there exists a scalar $\gamma \in A$, which does not depend on $s$ or on $v$, such that $f_{s t}^{\lambda} f_{t v}^{\lambda}=\gamma a_{s v}^{\lambda}$ plus a linear combination of more dominant terms. By Lemma 3.3(b) and (3.8), the coefficient of $f_{s v}^{\lambda}$ in $f_{s t}^{\lambda} f_{t v}^{\lambda}$ is equal to the coefficient of $a_{s v}^{\lambda}$ in $f_{s t}^{\lambda} f_{t v}^{\lambda}$, so this completes the proof.

We call $\left\{f_{s t}^{\lambda} \mid s, t \in T(\lambda)\right.$ and $\left.\lambda \in \Lambda\right\}$ the seminormal basis of $A$. This terminology is justified by Remark 3.13 below.

3.9. Corollary. Suppose that $A_{K}$ is a cellular algebra with a family of JM-elements which separate $T(\Lambda)$. Then $\gamma_{t} \neq 0$, for all $t \in T(\Lambda)$.

Proof. Suppose by way of contradiction that $\gamma_{t}=0$, for some $t \in T(\lambda)$ and $\lambda \in \Lambda$. Then, by Theorem 3.7, $f_{t t}^{\lambda} f_{u v}^{\mu}=0=f_{u v}^{\mu} f_{t t}^{\lambda}$, for all $u, v \in T(\mu), \mu \in \Lambda$. Therefore, $K f_{t t}^{\lambda}$ is a one dimensional nilpotent ideal of $A_{K}$, so $A_{K}$ is not semisimple. This contradicts Corollary 2.9, so we must have $\gamma_{t} \neq 0$ for all $t \in T(\Lambda)$.

Next, we use the basis $\left\{f_{\text {st }}^{\lambda}\right\}$ to identify the cell modules of $A$ as submodules of $A$.

3.10. Corollary. Suppose that $\lambda \in \Lambda$ and fix $s, t \in T(\lambda)$. Then

$$
C(\lambda) \cong f_{s t}^{\lambda} A_{K}=\operatorname{Span}\left\{f_{s v}^{\lambda} \mid v \in T(\lambda)\right\}
$$


Proof. As $f_{u v}^{\mu}=F_{u} a_{u v}^{\mu} F_{v}$, for $u, v \in T(\mu)$, the cell modules for the cellular bases $\left\{a_{u v}^{\lambda}\right\}$ and $\left\{f_{u v}^{\lambda}\right\}$ of $A_{K}$ coincide. Therefore, $C(\lambda)$ is isomorphic to the $A_{K}$-module $C(\lambda)^{\prime}$ which is spanned by the elements $\left\{f_{s u}^{\lambda}+A_{K}^{\lambda} \mid u \in T(\lambda)\right\}$.

On the other hand, if $u, v \in T(\mu)$, for $\mu \in \Lambda$, then $f_{s t}^{\lambda} f_{u v}^{\mu}=\delta_{t u} \gamma_{t} f_{s v}^{\lambda}$ by Theorem 3.7. Now $\gamma_{t} \neq 0$, by Corollary 3.9, so $\left\{f_{s v}^{\lambda} \mid v \in T(\lambda)\right\}$ is a basis of $f_{s t}^{\lambda} A_{K}$.

Finally, by Theorem 3.7 we have that $f_{s t}^{\lambda} A_{K} \cong C(\lambda)^{\prime}$, where the isomorphism is the linear extension of the map $f_{s v}^{\lambda} \mapsto f_{s v}^{\lambda}+A_{K}^{\lambda}$, for $v \in T(\lambda)$. Hence, $C(\lambda) \cong C(\lambda)^{\prime} \cong f_{s t}^{\lambda} A_{K}$, as required.

Recall that $\operatorname{rad} C(\lambda)$ is the radical of the bilinear form on $C(\lambda)$ and that $D(\lambda)=C(\lambda) / \operatorname{rad} C(\lambda)$.

Using Corollary 3.10 and Theorem 3.7, the basis $\left\{f_{s t}^{\lambda}\right\}$ gives an explicit decomposition of $A_{K}$ into a direct sum of cell modules. Abstractly this also follows from Corollary 2.9 and the general theory of cellular algebras because a cellular algebra is semisimple if and only if $C(\lambda)=D(\lambda)$, for all $\lambda \in \Lambda$; see [6], Theorem 3.4.

3.11. Corollary. Suppose that $A_{K}$ is a cellular algebra with a family of JM-elements which separate $T(\Lambda)$. Then $C(\lambda)=D(\lambda)$, for all $\lambda \in \Lambda$, and

$$
A_{K} \cong \bigoplus_{\lambda \in \Lambda} C(\lambda)^{\oplus|T(\lambda)|}
$$

Fix $s \in T(\lambda)$ and, for notational convenience, set $f_{t}^{\lambda}=f_{s t}^{\lambda}$ so that $C(\lambda)$ has basis $\left\{f_{t}^{\lambda} \mid t \in T(\lambda)\right\}$ by Corollary 3.10. Note that $f_{t}^{\lambda}=a_{t}^{\lambda}+\sum_{v \triangleright t} b_{v} a_{v}^{\lambda}$, for some $b_{v} \in K$, by Lemma 3.3(a).

For $\lambda \in \Lambda$ let $G(\lambda)=\operatorname{det}\left(\left\langle a_{s}^{\lambda}, a_{t}^{\lambda}\right\rangle_{\lambda}\right)_{s, t \in T(\lambda)}$ be the Gram determinant of the bilinear form $\langle,\rangle_{\lambda}$ on the cell module $C(\lambda)$. Note that $G(\lambda)$ is well-defined only up to multiplication by \pm 1 as we have not specified an ordering on the rows and columns of the Gram matrix.

3.12. Theorem. Suppose that $A_{K}$ is a cellular algebra with a family of JM-elements which separate $T(\Lambda)$. Let $\lambda \in \Lambda$ and suppose that $s, t \in T(\lambda)$. Then

$$
\left\langle f_{s}^{\lambda}, f_{t}^{\lambda}\right\rangle_{\lambda}=\left\langle a_{s}^{\lambda}, f_{t}^{\lambda}\right\rangle_{\lambda}= \begin{cases}\gamma_{t}, & \text { if } s=t \\ 0, & \text { otherwise }\end{cases}
$$

Consequently, $G(\lambda)=\prod_{t \in T(\lambda)} \gamma_{t}$.

Proof. By Theorem 3.7, $\left\{f_{s t}^{\lambda}\right\}$ is a cellular basis of $A_{K}$ and, by Corollary 3.10, we may take $\left\{f_{t}^{\lambda} \mid t \in T(\lambda)\right\}$ to be a basis of $C(\lambda)$. By Theorem 3.7 again, $f_{u s}^{\lambda} f_{t v}^{\lambda}=\delta_{s t} \gamma_{t} f_{u v}^{\lambda}$, so that $\left\langle f_{s}^{\lambda}, f_{t}^{\lambda}\right\rangle_{\lambda}=\delta_{s t} \gamma_{t}$ by Corollary 3.10 and the definition of the inner product on $C(\lambda)$. Using Proposition 3.4(b) and the associativity of the inner product on $C(\lambda)$, we see that

$$
\left\langle a_{s}^{\lambda}, f_{t}^{\lambda}\right\rangle_{\lambda}=\left\langle a_{s}^{\lambda}, f_{t}^{\lambda} F_{t}\right\rangle_{\lambda}=\left\langle a_{s}^{\lambda} F_{t}^{*}, f_{t}^{\lambda}\right\rangle_{\lambda}=\left\langle a_{s}^{\lambda} F_{t}, f_{t}^{\lambda}\right\rangle_{\lambda}=\left\langle f_{s}^{\lambda}, f_{t}^{\lambda}\right\rangle_{\lambda} .
$$

So we have proved the first claim in the statement of the theorem. 
Finally, the transition matrix between the two bases $\left\{a_{t}^{\lambda}\right\}$ and $\left\{f_{t}^{\lambda}\right\}$ of $C(\lambda)$ is unitriangular (when suitably ordered), so we have that

$$
G(\lambda)=\operatorname{det}\left(\left\langle a_{s}^{\lambda}, a_{t}^{\lambda}\right\rangle_{\lambda}\right)=\operatorname{det}\left(\left\langle f_{s}^{\lambda}, f_{t}^{\lambda}\right\rangle_{\lambda}\right)=\prod_{t \in T(\lambda)} \gamma_{t}
$$

as required.

3.13. Remark. Extending the bilinear forms $\langle,\rangle_{\lambda}$ to the whole of $A_{K}$ (using Corollary 3.11), we see that the seminormal basis $\left\{f_{s t}^{\lambda}\right\}$ is an orthogonal basis of $A_{K}$ with respect to this form.

In principle, we can use Theorem 3.12 to compute the Gram determinants of the cell modules of any cellular algebra $A$ which has a separable family of JM-elements. In practice, of course, we need to find formulae for the structure constants $\left\{\gamma_{t} \mid t \in T(\lambda)\right\}$ of the basis $\left\{f_{s t}^{\lambda}\right\}$. In all known examples, explicit formulae for $\gamma_{t}$ can be determined inductively once the actions of the generators of $A$ on the seminormal basis have been determined. In turn, the action of $A$ on its seminormal basis is determined by its action on the original cellular basis $\left\{a_{s t}^{\lambda}\right\}$. In effect, Theorem 3.12 gives an effective recipe for computing the Gram determinants of the cell modules of $A$.

By definition the scalars $\gamma_{t}$ are elements of the field $K$, for $t \in T(\lambda)$. Surprisingly, their product must belong to $R$.

3.14. Corollary. Suppose that $\lambda \in \Lambda$. Then $\prod_{t \in T(\lambda)} \gamma_{t} \in R$.

Proof. By definition, the inner products $\left\langle a_{s}^{\lambda}, a_{t}^{\lambda}\right\rangle_{\lambda}$ all belong to $R$, so $G(\lambda) \in R$. The result now follows from Theorem 3.12.

As $G(\lambda) \neq 0$ by Theorem 3.12 and Corollary 3.9 , it follows that each cell module is irreducible.

3.15. Corollary. Suppose that $\lambda \in \Lambda$. Then the cell module $C(\lambda)=D(\lambda)$ is an irreducible $A_{K}$-module.

We close this section by describing the primitive idempotents in $A_{K}$.

3.16. Theorem. Suppose that $A_{K}$ is a cellular algebra with a family of JM-elements which separate $T(\Lambda)$. Then

(a) If $t \in T(\lambda)$ and $\lambda \in \Lambda$ then $F_{t}=\frac{1}{\gamma_{t}} f_{t t}^{\lambda}$ and $F_{t}$ is a primitive idempotent in $A_{K}$.

(b) If $\lambda \in \Lambda$ then $F_{\lambda}=\sum_{t \in T(\lambda)} F_{t}$ is a primitive central idempotent in $A_{K}$.

(c) $\left\{F_{t} \mid t \in T(\Lambda)\right\}$ and $\left\{F_{\lambda} \mid \lambda \in \Lambda\right\}$ are complete sets of pairwise orthogonal idempotents in $A_{K}$; in particular,

$$
1_{A_{K}}=\sum_{\lambda \in \Lambda} F_{\lambda}=\sum_{t \in T(\Lambda)} F_{t}
$$


Proof. By Corollary 3.9, $\gamma_{t} \neq 0$ for all $t \in T(\lambda)$, so the statement of the theorem makes sense. Furthermore, $\frac{1}{\gamma_{t}} f_{t t}^{\lambda}$ is an idempotent by Theorem 3.7. By Corollary 3.15 the cell module $C(\lambda)$ is irreducible and by Corollary 3.10, $C(\lambda) \cong f_{t t}^{\lambda} A_{K}=F_{t} A_{K}$. Hence, $F_{t}$ is a primitive idempotent.

To complete the proof of (a) we still need to show that $F_{t}=\frac{1}{\gamma_{t}} f_{t t}^{\lambda}$. By Theorem 3.7 we can write $F_{t}=\sum_{v \in \Lambda} \sum_{x, y \in T(v)} r_{x y} f_{x y}^{v}$, for some $r_{x y} \in K$. Suppose that $u, v \in T(\mu)$, for some $\mu \in \Lambda$. Then, by Proposition 3.4 and Theorem 3.7,

$$
\delta_{v t} f_{u v}^{\mu}=f_{u v}^{\mu} F_{t}=\sum_{v \in \Lambda} \sum_{x, y \in T(\mu)} r_{x y} f_{u v}^{\mu} f_{x y}^{v}=\sum_{y \in T(\mu)} r_{v y} \gamma_{v} f_{u y}^{\mu} .
$$

By Corollary 3.9, $\gamma_{v} \neq 0$, so comparing both sides of this equation shows that

$$
r_{v y}= \begin{cases}\frac{1}{\gamma_{t}}, & \text { if } v=t=y \\ 0, & \text { otherwise }\end{cases}
$$

As $v$ is arbitrary we have $F_{t}=\frac{1}{\gamma_{t}} f_{t t}^{\lambda}$, as claimed.

This completes the proof of (a). Parts (b) and (c) now follow from (a) and the multiplication formula in Theorem 3.7.

3.17. Corollary. Suppose that $A_{K}$ is a cellular algebra with a family of JM-elements which separate $T(\Lambda)$. Then

$$
L_{i}=\sum_{t \in T(\Lambda)} \mathrm{c}_{t}(i) F_{t}
$$

and $\prod_{c \in \mathscr{C}(i)}\left(L_{i}-c\right)$ is the minimum polynomial for $L_{i}$ acting on $A_{K}$.

Proof. By part (c) of Theorem 3.16,

$$
L_{i}=L_{i} \sum_{t \in T(\Lambda)} F_{t}=\sum_{t \in T(\Lambda)} L_{i} F_{t}=\sum_{t \in T(\Lambda)} \mathrm{c}_{t}(i) F_{t}
$$

where the last equality follows from Proposition 3.4(c).

For the second claim, observe that $\prod_{c \in \mathscr{C}(i)}\left(L_{i}-c\right) \cdot f_{s t}^{\lambda}=0$ by Proposition 3.4(c), for all $\lambda \in \Lambda$ and all $s, t \in T(\lambda)$. If we omit the factor $\left(L_{i}-d\right)$, for some $d \in \mathscr{C}(i)$, then we can find an $s \in T(\mu)$, for some $\mu$, such that $\mathrm{c}_{s}(i)=d$ so that $\prod_{c \neq d}\left(L_{i}-c\right) F_{s} \neq 0$. Hence, $\prod_{c \in \mathscr{C}(i)}\left(L_{i}-c\right)$ is the minimum polynomial for the action of $L_{i}$ on $A_{K}$.

The examples at the end of section 2 show that the number of JM-elements is not uniquely determined. Nonetheless, we are able to characterize the subalgebra of $A_{K}$ which they generate when the JM-elements separate $T(\lambda)$. 
3.18. Corollary. Suppose that $A_{K}$ is a cellular algebra with a family of JM-elements which separate $T(\Lambda)$. Then $\left\{L_{1}, \ldots, L_{M}\right\}$ generate a maximal abelian subalgebra of $A_{K}$.

Proof. As the JM-elements commute, by definition, the subalgebra $\mathscr{L}_{K}$ of $A_{K}$ which they generate is certainly abelian. By Theorem 3.16 and Corollary $3.17, \mathscr{L}_{K}$ is the subalgebra of $A$ spanned by the primitive idempotents $\left\{F_{t} \mid t \in T(\Lambda)\right\}$. As the primitive idempotents of $A_{K}$ span a maximal abelian subalgebra of $A_{K}$, we are done.

\section{The non-separated case}

Up until now we have considered those cellular algebras $A_{K}$ which have a family of JM-elements which separate $T(\Lambda)$. By Corollary 2.9 the separation condition forces $A_{K}$ to be semisimple. In this section we still assume that $A=A_{R}$ has a family of JM-elements which separate $T(\Lambda)$ over $R$ but rather than studying the semisimple algebra $A_{K}$ we extend the previous constructions to non-separated algebras over a field.

In this section let $R$ be a discrete valuation ring with maximal ideal $\pi$. We assume that $A_{R}$ has a family of JM-elements which separate $T(\Lambda)$ over $R$.

Let $K$ be the field of fractions of $R$. Then $A_{K}$ is semisimple by Corollary 2.9 and all of the results of the previous section apply to $A_{K}$. Let $k=R / \pi$ be the residue field of $K$. Then $A_{k}=A \otimes_{R} k$ is a cellular algebra with cellular basis given by the image of the cellular basis of $A$ in $A_{k}$. We abuse notation and write $\left\{a_{s t}^{\lambda}\right\}$ for the cellular bases of all three algebras $A=A_{R}, A_{K}$ and $A_{k}$. It should always be clear from the context which algebra these elements belong to at any given time.

In general, the JM-elements will not separate $T(\Lambda)$ over $k$, so the arguments of the previous section do not necessarily apply to the algebra $A_{k}$.

If $r \in R$ let $\bar{r}=r+\pi$ be its image in $k=R / \pi$. More generally, if $a=\sum r_{s t} a_{s t}^{\lambda} \in A_{R}$ then we set $\bar{a}=\sum \overline{r_{s t}} a_{s t}^{\lambda} \in A_{k}$.

Observe that $c-c^{\prime}$ is invertible in $R$ whenever $\bar{c} \neq \bar{c}^{\prime}$ and $c, c^{\prime} \in \mathscr{C}=\bigcup_{i=1}^{M} \mathscr{C}(i)$.

If $1 \leqq i \leqq M$ and $t \in T(\lambda)$ define the residue of $i$ at $t$ to be $\mathrm{r}_{t}(i)=\overline{\mathrm{c}_{t}(i)}$. By 2.4 the action of the JM-elements on $A_{k}$ is given by

$$
a_{s t}^{\lambda} L_{i} \equiv \mathrm{r}_{t}(i) a_{s t}^{\lambda}+\sum_{v \triangleright t} r_{t v} a_{s v}^{\lambda} \quad\left(\bmod A_{k}^{\lambda}\right)
$$

where $r_{t v} \in k$ (and otherwise the notation is as in 2.4). There is an analogous formula for the action of $L_{i}$ on $a_{s t}^{\lambda}$ from the left.

We use residues modulo $\pi$ to define equivalence relations on $T(\Lambda)$ and on $\Lambda$.

4.1. Definition (Residue classes and linkage classes). (a) Suppose that $s, t \in T(\Lambda)$. Then $s$ and $t$ are in the same residue class, and we write $s \approx t$, if $\mathrm{r}_{s}(i)=\mathrm{r}_{t}(i)$, for $1 \leqq i \leqq M$. 
(b) Suppose that $\lambda, \mu \in \Lambda$. Then $\lambda$ and $\mu$ are residually linked, and we write $\lambda \sim \mu$, if there exist elements $\lambda_{0}=\lambda, \lambda_{1}, \ldots, \lambda_{r}=\mu$ and elements $s_{j}, t_{j} \in T\left(\lambda_{j}\right)$ such that $s_{j-1} \approx t_{j}$, for $i=1, \ldots, r$.

It is easy to see that $\approx$ is an equivalence relation on $T(\Lambda)$ and that $\sim$ is an equivalence relation on $\Lambda$. If $s \in T(\Lambda)$ let $\mathbb{T}_{s} \in T(\Lambda) / \approx$ be its residue class. If $\mathbb{T}$ is a residue class let $\mathbb{T}(\lambda)=\mathbb{T} \cap T(\lambda)$, for $\lambda \in \Lambda$. By 2.6 , the residue classes $T(\Lambda) / \approx$ parameterize the irreducible $\mathscr{L}_{k}$-modules.

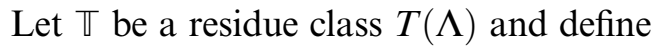

$$
F_{\mathbb{T}}=\sum_{t \in \mathbb{T}} F_{t}
$$

By definition, $F_{\mathbb{T}}$ is an element of $A_{K}$. We claim that, in fact, $F_{\mathbb{T}} \in A_{R}$.

The following argument is an adaptation of Murphy's proof of [18], Theorem 2.1.

4.2. Lemma. Suppose that $\mathbb{T}$ is a residue equivalence class in $T(\Lambda)$. Then $F_{\mathbb{T}}$ is an idempotent in $A_{R}$.

Proof. We first note that $F_{\square}$ is an idempotent in $A_{K}$ because it is a linear combination of orthogonal idempotents by Theorem 3.16(a). The hard part is proving that $F_{\mathbb{T}} \in A_{R}$.

Fix an element $t \in \mathbb{T}(\mu)$, where $\mu \in \Lambda$, and define

$$
F_{t}^{\prime}=\prod_{i=1}^{M} \prod_{\substack{c \in \mathscr{C} \\ \bar{c} \neq r_{t}(i)}} \frac{L_{i}-c}{\mathrm{c}_{t}(i)-c}
$$

Then $F_{t}^{\prime} \in A_{R}$ since, by assumption, $\mathrm{c}_{t}(i)-c$ is invertible in $R$ whenever $\mathrm{r}_{t}(i) \neq \bar{c}$. Observe that the numerator of $F_{t}^{\prime}$ depends only on $\mathbb{T}$ whereas the denominator $d_{t}=\prod_{i=1}^{M} \prod_{\bar{c} \neq \mathrm{r}_{t}(i)}\left(\mathrm{c}_{t}(i)-c\right)$ of $F_{t}^{\prime}$ depends on $t$. Let $s \in T(\lambda)$. Then, by Proposition 3.4(d) and Theorem 3.16(a),

$$
F_{t}^{\prime} F_{s}= \begin{cases}\frac{d_{s}}{d_{t}} F_{s}, & \text { if } s \in \mathbb{T} \\ 0, & \text { otherwise }\end{cases}
$$

Consequently, $F_{t}^{\prime}=\sum_{\lambda \in \Lambda} \sum_{s \in \mathbb{T}(\lambda)} \frac{d_{s}}{d_{t}} F_{s}$, by Theorem 3.16(c).

Now, if $s \in \mathbb{T}(\lambda)$ then $d_{s} \equiv d_{t}(\bmod \pi)$ since $s \approx t$. Therefore, $1-\frac{d_{s}}{d_{t}}$ is an element of $\pi$. Let $e_{s} \in R$ be the denominator of $F_{s}$ and choose $N$ maximal such that $e_{s} \in \pi^{N}$, for all $s \in \mathbb{T}$. Then $\left(1-\frac{d_{s}}{d_{t}}\right)^{N} \frac{1}{e_{s}} \in R$, so that $\left(1-\frac{d_{s}}{d_{t}}\right)^{N} F_{s} \in A_{R}$, for all $s \in \mathbb{T}$. We now compute 


$$
\begin{aligned}
\left(F_{\mathbb{T}}-F_{t}^{\prime}\right)^{N} & =\left(\sum_{\lambda \in \Lambda} \sum_{s \in \mathbb{T}(\lambda)}\left(1-\frac{d_{s}}{d_{t}}\right) F_{s}\right)^{N} \\
& =\sum_{\lambda \in \Lambda} \sum_{s \in \mathbb{T}(\lambda)}\left(1-\frac{d_{s}}{d_{t}}\right)^{N} F_{s},
\end{aligned}
$$

where the last line follows because the $F_{s}$ are pairwise orthogonal idempotents in $A_{K}$. Therefore, $\left(F_{\mathbb{J}}-F_{t}^{\prime}\right)^{N} \in A_{R}$.

To complete the proof we evaluate $\left(F_{\mathbb{T}}-F_{t}^{\prime}\right)^{N}$ directly. First, by Theorem 3.16(a),

$$
F_{t}^{\prime} F_{\mathbb{T}}=\sum_{\lambda \in \Lambda} \sum_{s \in \mathbb{T}(\lambda)} \frac{d_{s}}{d_{t}} F_{s} F_{\mathbb{T}}=\sum_{\lambda \in \Lambda} \sum_{s \in \mathbb{T}(\lambda)} \frac{d_{s}}{d_{t}} F_{s}=F_{t}^{\prime} .
$$

Similarly, $F_{\mathbb{V}} F_{t}^{\prime}=F_{t}^{\prime}$. Hence, using the binomial theorem, we have

$$
\begin{aligned}
\left(F_{\mathbb{T}}-F_{t}^{\prime}\right)^{N} & =\sum_{i=0}^{N}(-1)^{i}\left(\begin{array}{c}
N \\
i
\end{array}\right)\left(F_{t}^{\prime}\right)^{i} F_{\mathbb{T}}^{N-i} \\
& =F_{\mathbb{T}}+\sum_{i=1}^{N}(-1)^{i}\left(\begin{array}{c}
N \\
i
\end{array}\right)\left(F_{t}^{\prime}\right)^{i} \\
& =F_{\mathbb{T}}+\left(1-F_{t}^{\prime}\right)^{N}-1 .
\end{aligned}
$$

Hence, $F_{\mathbb{T}}=\left(F_{\mathbb{T}}-F_{t}^{\prime}\right)^{N}-\left(1-F_{t}^{\prime}\right)^{N}+1 \in A_{R}$, as required.

By the lemma, $F_{\mathbb{T}} \in A_{R}$. Therefore, we can reduce $F_{\mathbb{T}}$ modulo $\pi$ to obtain an element of $A_{k}$. Let $G_{\mathbb{T}}=\overline{F_{\mathbb{T}}} \in A_{k}$ be the reduction of $F_{\mathbb{T}}$ modulo $\pi$. Then $G_{\mathbb{}}$ is an idempotent in $A_{k}$.

Recall that if $s \in T(\Lambda)$ then $\mathbb{T}_{s}$ is its residue class.

4.3. Definition. Let $\mathbb{T}$ be a residue class of $T(\Lambda)$.

(a) Suppose that $s, t \in T(\lambda)$. Define $g_{s t}^{\lambda}=G_{\mathbb{J}_{s}} a_{s t}^{\lambda} G_{\mathbb{J}_{t}} \in A_{k}$.

(b) Suppose that $\Gamma \in \Lambda / \sim$ is a residue linkage class in $\Lambda$. Let $A_{k}^{\Gamma}$ be the subspace of $A_{k}$ spanned by $\left\{g_{s t}^{\lambda} \mid s, t \in T(\lambda)\right.$ and $\left.\lambda \in \Gamma\right\}$.

Note that $G_{\mathbb{T}}^{*}=G_{\mathbb{T}}$ and that $\left(g_{s t}^{\lambda}\right)^{*}=g_{t s}^{\lambda}$, for all $s, t \in T(\lambda)$ and $\lambda \in \Lambda$. By Theorem 3.16, if $\mathbb{S}$ and $\mathbb{T}$ are residue classes in $T(\Lambda)$ then $G_{\mathbb{S}} G_{\mathbb{T}}=\delta_{\mathbb{S} \mathbb{T}} G_{\mathbb{}}$.

4.4. Proposition. Suppose that $s, t \in T(\lambda)$, for some $\lambda \in \Lambda$, that $u \in T(\Lambda)$ and fix $i$ with $1 \leqq i \leqq M$. Let $\mathbb{T} \in \mathbb{T}(\Lambda) / \approx$. Then, in $A_{k}$,
(a) $L_{i} g_{s t}^{\lambda}=\mathrm{r}_{s}(i) g_{s t}^{\lambda}+\sum_{u \triangleright s} \mathrm{r}_{u} g_{u t}^{\lambda} \bmod A^{\lambda}$,
(c) $G_{\mathbb{}} g_{s t}^{\lambda}=\delta_{\mathbb{U}_{s} \rrbracket} g_{s t}^{\lambda}$,
(b) $g_{s t}^{\lambda} L_{i}=\mathrm{r}_{t}(i) g_{s t}^{\lambda}+\sum_{v \triangleright t} \mathrm{r}_{v} g_{s v}^{\lambda} \bmod A^{\lambda}$,
(d) $g_{s t}^{\lambda} G_{\mathbb{T}}=\delta_{\mathbb{}} g_{t} g_{s t}^{\lambda}$ 
We can now generalize the seminormal basis of the previous section to the algebra $A_{k}$.

4.5. Theorem. Suppose that $A_{R}$ has a family of JM-elements which separate $T(\Lambda)$ over $R$.

(a) $\left\{g_{s t}^{\lambda} \mid s, t \in T(\lambda)\right.$ and $\left.\lambda \in \Lambda\right\}$ is a cellular basis of $A_{k}$.

(b) Let $\Gamma$ be a residue linkage class of $\Lambda$. Then $A_{k}^{\Gamma}$ is a cellular algebra with cellular basis $\left\{g_{s t}^{\lambda} \mid s, t \in T(\lambda)\right.$ and $\left.\lambda \in \Gamma\right\}$.

(c) The residue linkage classes decompose $A_{k}$ into a direct sum of cellular subalgebras; that is,

$$
A_{k}=\underset{\Gamma \in \Lambda / \sim}{\bigoplus_{k}^{\Gamma}}
$$

Proof. Let $\Gamma$ be a residue linkage class in $\Lambda$ and suppose that $\lambda \in \Gamma$. Then, exactly as in the proof of Lemma 3.3(a), we see that if $s, t \in T(\lambda)$ then $g_{s t}^{\lambda}=a_{s t}^{\lambda}$ plus a linear combination of more dominant terms. Therefore, the elements $\left\{g_{s t}^{\lambda}\right\}$ are linearly independent because $\left\{a_{s t}^{\lambda}\right\}$ is a basis of $A_{k}$. Hence, $\left\{g_{s t}^{\lambda}\right\}$ is a basis of $A_{k}$. We prove the remaining statements in the theorem simultaneously.

Suppose that $\lambda, \mu \in \Lambda$ and that $s, t \in \mathbb{T}(\lambda)$ and $u, v \in \mathbb{T}(\mu)$. Then

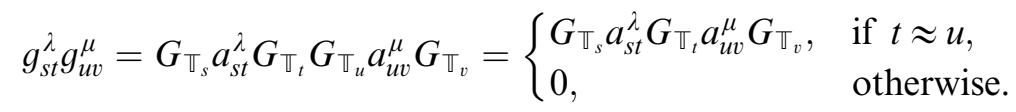

Observe that $t \approx u$ only if $\lambda \sim \mu$. Suppose then that $\lambda \sim \mu$ and let $\Gamma$ be the residue linkage class in $\Lambda$ which contains $\lambda$ and $\mu$. Then, because $\left\{a_{w x}^{v}\right\}$ is a cellular basis of $A_{k}$, we can write

$$
a_{s t}^{\lambda} G_{\mathbb{T}_{t}} a_{u v}^{\mu}=\sum_{\substack{v \in \Lambda \\ v \geqq \lambda, v \geqq \mu}} \sum_{\substack{w, x \in T(v) \\ v \unrhd s, x \unrhd v}} r_{w x} g_{w x}^{v},
$$

for some $r_{w x} \in k$ such that if $v=\lambda$ then $r_{w x} \neq 0$ only if $w=s$, and if $v=\mu$ then $r_{w x} \neq 0$ only if $x=v$. Therefore, using Proposition 4.4, we have

$$
\begin{aligned}
g_{s t}^{\lambda} g_{u v}^{\mu} & =\sum_{\substack{v \in \Lambda \\
v \geqq \lambda, v \geqq \mu}} \sum_{\substack{w, x \in T(v) \\
w \unrhd s, x \unrhd v}} r_{w x} G_{\mathbb{T}_{s}} g_{w x}^{v} G_{\mathbb{T}_{v}} \\
& =\sum_{\substack{v \in \Gamma \\
v \geqq \lambda, v \geqq \mu}} \sum_{\substack{w, x \in T(v) \\
w \unrhd s, x \unrhd v}} r_{w x} g_{w x}^{v} .
\end{aligned}
$$

Consequently, we see that if $\lambda \sim \mu \in \Gamma$ then $g_{s t}^{\lambda} g_{u v}^{\mu} \in A_{k}^{\Gamma}$; otherwise, $g_{s t}^{\lambda} g_{u v}^{\mu}=0$. All of the statements in the theorem now follow.

Arguing as in the proof of Theorem 3.16(a) it follows that $G_{\mathbb{}}=\sum r_{s t} g_{s t}^{\lambda}$, where $r_{s t}$ is non-zero only if $s, t \in \mathbb{T}(\lambda)$ for some $\lambda \in \Lambda$. 
We are not claiming in Theorem 4.5 that the subalgebras $A_{k}^{\Gamma}$ of $A_{k}$ are indecomposable. We call the indecomposable two-sided ideals of $A_{k}$ the blocks of $A_{k}$. It is a general fact that each irreducible module of an algebra is a composition factor of a unique block, so the residue linkage classes induce a partition of the set of irreducible $A_{k}$-modules. By the general theory of cellular algebras, all of the composition factors of a cell module are contained in the same block; see [6], 3.9.8, or [15], Cor. 2.22. Hence, we have the following.

4.6. Corollary. Suppose that $A_{R}$ has a family of JM-elements which separate $T(\Lambda)$ over $R$ and that $\lambda, \mu \in \Lambda$. Then $C(\lambda)$ and $C(\mu)$ are in the same block of $A_{k}$ only if $\lambda \sim \mu$.

Let $\Gamma \in \Lambda / \sim$ be a residue linkage class. Then $\sum_{\lambda \in \Gamma} F_{\lambda} \in A_{R}$ by Lemma 4.2 and Theorem $3.16(\mathrm{~b})$. Set $G_{\Gamma}=\overline{\sum_{\lambda \in \Gamma} F_{\lambda}} \in A_{k}$. The following result is now immediate from Theorem 4.5 and Theorem 3.16. over $R$.

4.7. Corollary. Suppose that $A_{R}$ has a family of JM-elements which separate $T(\Lambda)$

(a) Let $\Gamma$ be a residue linkage class. Then $G_{\Gamma}$ is a central idempotent in $A_{k}$ and the identity element of the subalgebra $A_{k}^{\Gamma}$. Moreover,

$$
A_{k}^{\Gamma}=G_{\Gamma} A_{k} G_{\Gamma} \cong \operatorname{End}_{A_{k}}\left(A_{k} G_{\Gamma}\right) .
$$

(b) $\left\{G_{\Gamma} \mid \Gamma \in \Lambda / \sim\right\}$ and $\left\{G_{\mathbb{U}} \mid \mathbb{T} \in T(\Lambda) / \approx\right\}$ are complete sets of pairwise orthogonal idempotents of $A_{k}$. In particular,

$$
1_{A_{k}}=\sum_{\Gamma \in \Lambda / \sim} G_{\Gamma}=\sum_{\mathbb{T} \in T(\Lambda) / \approx} G_{\mathbb{T}}
$$

Observe that the right ideals $G_{\mathbb{}} A_{k}$ are projective $A_{k}$-modules, for all $\mathbb{T} \in T(\Lambda) / \approx$. Of course, these modules need not (and, in general, will not) be indecomposable.

Let $\mathscr{R}(i)=\{\bar{c} \mid c \in \mathscr{C}(i)\}$, for $1 \leqq i \leqq M$. If $\mathbb{T}$ is a residue class in $T(\Lambda)$ then we set $\mathrm{r}_{\mathbb{T}}(i)=\mathrm{r}_{t}(i)$, for $t \in \mathbb{T}$ and $1 \leqq i \leqq M$.

4.8. Corollary. Suppose that $A_{R}$ has a family of JM-elements which separate $T(\Lambda)$ over R. Then

$$
L_{i}=\sum_{\mathbb{T} \in T(\Lambda) / \approx} \mathrm{r}_{\mathbb{T}}(i) G_{\mathbb{T}}
$$

Proof. That $L_{i}=\sum_{\mathbb{T} \in T(\Lambda) / \approx} \mathrm{r}_{\mathbb{T}}(i) G_{\mathbb{T}}$ follows from Corollary 4.7(b) and Proposition 4.4 .

As our final general result we note that the new cellular basis of $A_{k}$ gives us a new 'not quite orthogonal' basis for the cell modules of $A_{k}$. Given $\lambda \in \Lambda$ fix $s \in T(\lambda)$ and define $g_{t}^{\lambda}=g_{s t}^{\lambda}+A_{k}^{\lambda}$ for $t \in T(\lambda)$. 
4.9. Proposition. Suppose that $A_{R}$ has a family of JM-elements which separate $T(\Lambda)$ over $R$. Then $\left\{g_{t}^{\lambda} \mid t \in T(\lambda)\right\}$ is a basis of $C(\lambda)$. Moreover, if $t, u \in T(\lambda)$ then

$$
\left\langle g_{t}^{\lambda}, g_{u}^{\lambda}\right\rangle_{\lambda}= \begin{cases}\left\langle a_{t}^{\lambda}, g_{u}^{\lambda}\right\rangle_{\lambda}, & \text { if } t \approx u, \\ 0, & \text { if } t \approx u .\end{cases}
$$

Proof. That $\left\{g_{t}^{\lambda} \mid t \in T(\lambda)\right\}$ is a basis of $C(\lambda)$ follows from Theorem 4.5 and the argument of Lemma 3.3(a). For the second claim, if $t, u \in T(\lambda)$ then

$$
\left\langle g_{t}^{\lambda}, g_{u}^{\lambda}\right\rangle_{\lambda}=\left\langle a_{t}^{\lambda} G_{\mathbb{W}_{t}}, g_{u}^{\lambda}\right\rangle_{\lambda}=\left\langle a_{t}^{\lambda}, g_{u}^{\lambda} G_{\mathbb{\varpi}_{t}}\right\rangle_{\lambda}
$$

by the associativity of the inner product since $G_{\mathbb{T}_{t}}^{*}=G_{\mathbb{V}_{t}}$. The result now follows from Proposition 4.4(d).

In the semisimple case Theorem 3.12 reduces the Gram determinant of a cell module to diagonal form. This result reduces it to block diagonal form. Murphy has considered this block decomposition of the Gram determinant for the Hecke algebras of type $A$ [20].

We now apply the results of this section to give a basis for the blocks of several of the algebras considered in section 2 .

4.10. Theorem. Let $k$ be a field and suppose that $A_{R}$ is one of the following algebras:

(a) the group algebra $R \Im_{n}$ of the symmetric group;

(b) the Hecke algebra $\mathscr{H}_{R, q}\left(\Xi_{n}\right)$ of type A;

(c) the Ariki-Koike algebra $\mathscr{H}_{R, q, u}$ with $q \neq 1$;

(d) the degenerate Ariki-Koike algebra $\mathscr{H}_{R, v}$.

Then $A$ has a family of JM-elements which separate $T(\Lambda)$ over $R$ and Theorem 4.5 gives a basis for the block decomposition of $A_{k}$ into a direct sum of indecomposable subalgebras.

The cellular bases and the families of JM-elements for each of these algebras are given in the examples of Section 2. As $k \Im_{n} \cong \mathscr{H}_{k, 1}\left(\mathfrak{\Xi}_{n}\right)$, we use the Murphy basis for the symmetric group. Note that the Hecke algebras of type $A$ should not be considered as the special case $r=1$ of the Ariki-Koike algebras because the JM-elements that we use for these two algebras are different. Significantly, for the Ariki-Koike case we must assume that $q \neq 1$ as the JM-elements that we use do not separate $T(\Lambda)$ over $R$ when $q=1$.

Before we can begin proving this result we need to describe how to choose a modular system $(R, K, k)$ for each of the algebras above. In all cases we start with a field $k$ and a non-zero element $q \in k$ and we let $R$ be the localization of the Laurent polynomial ring $k\left[t, t^{-1}\right]$ at the maximal ideal generated by $(q-t)$. Then $R$ is a discrete valuation ring with maximal ideal $\pi$ generated by the image of $(q-t)$ in $R$. By construction, $k \cong R / \pi$ and $t$ is sent to $q$ by the natural map $R \rightarrow k=R / \pi$. Let $K$ be the field of fractions of $R$. 
First consider the case of the Hecke algebra $\mathscr{H}_{k, q}\left(\Xi_{n}\right)$. As we have said, this includes the symmetric group as the special case $q=1$. We take $A_{R}=\mathscr{H}_{R, t}\left(\Xi_{n}\right), A_{K}=\mathscr{H}_{K, t}\left(\Xi_{n}\right)$, and $A_{k}=\mathscr{H}_{R, t}\left(\Xi_{n}\right) \bigotimes_{R} k$. Then $\mathscr{H}_{K, t}\left(\Xi_{n}\right)$ is semisimple and $\mathscr{H}_{k, q}\left(\Xi_{n}\right) \cong \mathscr{H}_{R, t}\left(\Theta_{n}\right) \bigotimes_{R} k$.

Next, consider the Ariki-Koike algebra $\mathscr{H}_{k, q, u}$ with parameters $q \neq 0,1$ and $\boldsymbol{u}=\left(u_{1}, \ldots, u_{m}\right) \in k^{m}$. Let $v_{s}=u_{s}+(q-t)^{n s}$, for $s=1, \ldots, m$, and set $\boldsymbol{v}=\left(v_{1}, \ldots, v_{m}\right)$. We consider the triple of algebras $A_{R}=\mathscr{H}_{R, t, v}, A_{K}=\mathscr{H}_{K, t, \boldsymbol{v}}$ and $A_{k}=\mathscr{H}_{k, q, \boldsymbol{u}}$. Once again, $A_{K}$ is semisimple and $A_{k} \cong A_{R} \otimes_{R} k$. The case of the degenerate Ariki-Koike algebras is similar and we leave the details to the reader.

The indexing set $\Lambda$ for each of the algebras considered in Theorem 4.10 is the set of $m$-multipartitions of $n$, where we identify the set of 1-multipartitions with the set of partitions. If $\lambda$ is an $m$-multipartition let $[\lambda]$ be the diagram of $\lambda$; that is,

$$
[\lambda]=\left\{(s, i, j) \mid 1 \leqq s \leqq r \text { and } 1 \leqq j \leqq \lambda_{i}^{(s)}\right\}
$$

Given a node $x=(s, i, j) \in[\lambda]$ we define its content to be

$$
\mathrm{c}(x)= \begin{cases}{[j-i]_{t},} & \text { if } A_{R}=\mathscr{H}_{R, t}\left(\Xi_{n}\right), \\ v_{s} t^{j-i}, & \text { if } A_{R}=\mathscr{H}_{R, t, v} \\ v_{s}+(j-i), & \text { if } A_{R}=\mathscr{H}_{R, \boldsymbol{v}}\end{cases}
$$

We set $\mathscr{C}_{\lambda}=\{\mathrm{c}(x) \mid x \in[\lambda]\}$ and $\mathscr{R}_{\lambda}=\{\overline{\mathrm{c}(x)} \mid x \in[\lambda]\}$.

Unravelling the definitions, it is easy to see, for each of the algebras that we are considering, that if $\lambda \in \Lambda$ and $t \in T(\lambda)$ then $\mathscr{C}_{\lambda}=\left\{\mathrm{c}_{t}(i) \mid 1 \leqq i \leqq M\right\}$.

To prove Theorem 4.10 we need to show that the residue linkage classes correspond to the blocks of each of the algebras above. Hence, Theorem 4.10 is a corollary of the following proposition.

4.11. Proposition. Let $A$ be one of the algebras considered in Theorem 4.10. Suppose that $\lambda, \mu \in \Lambda$. The following are equivalent:

(a) $C(\lambda)$ and $C(\mu)$ belong to the same block of $A_{k}$.

(b) $\lambda \sim \mu$.

(c) $\mathscr{R}_{\lambda}=\mathscr{R}_{\mu}$.

Proof. First suppose that $C(\lambda)$ and $C(\mu)$ are in the same block. Then $\lambda \sim \mu$ by Corollary 4.6, so that (a) implies (b). Next, if (b) holds then, without loss of generality, there exist $s \in T(\lambda)$ and $t \in T(\mu)$ with $s \approx t$; however, then $\mathscr{R}_{\lambda}=\mathscr{R}_{\mu}$. So, (b) implies (c). The implication '(c) implies (a)' is the most difficult, however, the blocks of all of the algebras that we are considering have been classified and the result can be stated uniformly by saying that the cell modules $C(\lambda)$ and $C(\mu)$ belong to the same block if and only if $\mathscr{R}_{\lambda}=\mathscr{R}_{\mu}$; see 
[14] for $\mathscr{H}_{k, q}\left(\Xi_{n}\right)$ and the Ariki-Koike algebras, and [3] for the degenerate Ariki-Koike algebras. Therefore, (a) and (c) are equivalent. This completes the proof.

As a consequence we obtain the block decomposition of the Schur algebras. Let $\Lambda_{m, n}$ be the set of $m$-multipartitions of $n$ and let $S_{R, t, v}\left(\Lambda_{m, n}\right)$ be the corresponding cyclotomic $q$-Schur algebra [4], where $t$ and $\boldsymbol{v}$ are as above.

4.12. Corollary. Let $k$ be a field and suppose that $A$ is one of the following k-algebras:

(a) the q-Schur algebra $S_{R, q}(n)$;

(b) the cyclotomic $q$-Schur $S_{R, t, v}\left(\Lambda_{m, n}\right)$ algebra with $q \neq 1$.

Then $A$ has a family of JM-elements which separate $T(\Lambda)$ over $R$ and Theorem 4.5 gives a basis for the block decomposition of $A_{k}$ into a direct sum of indecomposable subalgebras.

Proof. Once again it is enough to show that two cell modules $C(\lambda)$ and $C(\mu)$ belong to the same block if and only if $\lambda \sim \mu$. By Schur-Weyl duality, the blocks of $S_{k, q}(n)$ are in bijection with the blocks of $\mathscr{H}_{k, q}(n)([15], 5.37-5.38)$ and the blocks of $S_{k, q, u}\left(\Lambda_{m, n}\right)$ are in bijection with the blocks of $\mathscr{H}_{k, q, u}([16]$, Theorem 5.5). Hence the result follows from Proposition 4.11 .

It is well known for each algebra $A$ in Theorem 4.10 that the symmetric polynomials in the JM-elements belong to the centre of $A$. As our final result we show that there is a uniform explanation of this fact. If $A$ is an algebra we let $Z(A)$ be its centre.

4.13. Proposition. Suppose that $A$ has a family of JM-elements which separate $T(\Lambda)$ over $R$ and that for $\lambda \in \Lambda$ there exist scalars $\mathrm{c}_{\lambda}(i)$, for $1 \leqq i \leqq M$, such that

$$
\left\{\mathrm{c}_{\lambda}(i) \mid 1 \leqq i \leqq M\right\}=\left\{\mathrm{c}_{t}(i) \mid 1 \leqq i \leqq M\right\}
$$

for any $t \in T(\lambda)$. Then any symmetric polynomial in $L_{1}, \ldots, L_{M}$ belongs to the centre of $A_{k}$.

Proof. Suppose that $X_{1}, \ldots, X_{M}$ are indeterminates over $R$ and let $p\left(X_{1}, \ldots, X_{M}\right) \in R\left[X_{1}, \ldots, X_{M}\right]$ be a symmetric polynomial. Recall that $L_{i}=\sum_{t} \mathrm{c}_{t}(i) F_{t}$ in
$A_{K}$, by Corollary 3.17 . Therefore,

$$
p\left(L_{1}, \ldots, L_{M}\right)=\sum_{t \in T(\Lambda)} p\left(c_{t}(1), \ldots, c_{t}(M)\right) F_{t}=\sum_{\lambda \in \Lambda} p\left(c_{\lambda}(1), \ldots, c_{\lambda}(M)\right) F_{\lambda}
$$

The first equality follows because the $F_{t}$ are pairwise orthogonal idempotents by Theorem 3.16. By Theorem 3.16(c) the centre of $A_{K}$ is spanned by the elements $\left\{F_{\lambda} \mid \lambda \in \Lambda\right\}$, so this shows that $p\left(L_{1}, \ldots, L_{M}\right)$ belongs to the centre of $A_{K}$. However, $p\left(L_{1}, \ldots, L_{M}\right)$ belongs to $A_{R}$ so, in fact, $p\left(L_{1}, \ldots, L_{M}\right)$ belongs to the centre of $A_{R}$. Now, $\overline{Z\left(A_{R}\right)}$ is contained in the centre of $A_{k}$ and any symmetric polynomial over $k$ can be lifted to a symmetric polynomial over $R$. Thus, it follows that the symmetric polynomials in the JM-elements of $A_{k}$ are central in $A_{k}$. 
All of the algebras in Theorem 4.10 satisfy the conditions of the proposition because, using the notation above, if $t \in T(\lambda)$ then $\mathscr{C}_{\lambda}=\left\{\mathrm{c}_{t}(i) \mid 1 \leqq i \leqq M\right\}$ for any of these algebras. Notice, however, that the (cyclotomic) Schur algebras considered in section 2 and the Brauer and BMW algebras do not satisfy the assumptions of Proposition 4.13.

Acknowledgements. I thank Marcos Soriano for many discussions about seminormal forms of Hecke algebras and for his detailed comments and suggestions on this paper. This paper also owes a debt to Gene Murphy as he pioneered the use of the Jucys-Murphy elements in the representation theory of symmetric groups and Hecke algebras.

\section{References}

[1] R. M. Adin, F. Brenti, and Y. Roichman, A unified construction of Coxeter group representations (I), Adv. Appl. Math., to appear.

[2] S. Ariki, A. Mathas, and H. Rui, Cyclotomic Nazarov-Wenzl algebras, Special issue in honour of George Lusztig, Nagoya J. Math. 182 (2006), 47-134.

[3] J. Brundan, Centers of degenerate cyclotomic Hecke algebras, parabolic category $O$, preprint 2006, math.RT/0607717.

[4] R. Dipper, G. James, and A. Mathas, Cyclotomic q-Schur algebras, Math. Z. 229 (1999), 385-416.

[5] J. Enyang, Specht modules and semisimplicity criteria for the Brauer and Birman-Murakami-Wenzl algebras, preprint 2005, math.RT/0705.4142.

[6] J. J. Graham and G. I. Lehrer, Cellular algebras, Invent. Math. 123 (1996), 1-34.

[7] T. Halverson and A. Ram, Characters of algebras containing a Jones basic construction: the Temperley-Lieb, Okasa, Brauer, and Birman-Wenzl algebras, Adv. Math. 116 (1995), 263-321.

[8] P. N. Hoefsmit, Representations of Hecke algebras of finite groups with BN-pairs of classical type, $\mathrm{PhD}$ thesis, University of British Columbia, 1979.

[9] G. D. James and A. Mathas, A q-analogue of the Jantzen-Schaper theorem, Proc. Lond. Math. Soc. (3) 74 (1997), 241-274.

[10] G. D. James and A. Mathas, The Jantzen sum formula for cyclotomic $q$-Schur algebras, Trans. Amer. Math. Soc. 352 (2000), 5381-5404.

[11] A. A. Jucys, On the Young operators of the symmetric groups, Lithuanian Phys. J. 11 (1971), 1-10.

[12] A. S. Kleshchev, Lectures on linear and projective representations of symmetric groups, CUP, 2005.

[13] R. Leduc and A. Ram, A ribbon Hopf algebra approach to the irreducible representations of centralizer algebras: the Brauer, Birman-Wenzl, and type A Iwahori-Hecke algebras, Adv. Math. 125 (1997), $1-94$.

[14] S. Lyle and A. Mathas, Blocks of cyclotomic Hecke algebras, Adv. Math. 216 (2007), 854-878.

[15] A. Mathas, Hecke algebras and Schur algebras of the symmetric group, Univ. Lect. Notes 15, Amer. Math. Soc., 1999.

[16] A. Mathas, The representation theory of the Ariki-Koike and cyclotomic $q$-Schur algebras, in: Representation theory of algebraic groups and quantum groups, Adv. Stud. Pure Math. 40, Math. Soc. Japan, Tokyo (2004), 261-320.

[17] G. E. Murphy, A new construction of Young's semi-normal representation of the symmetric groups, J. Algebra 69 (1981), 287-297.

[18] G. E. Murphy, The idempotents of the symmetric group and Nakayama's conjecture, J. Algebra 81 (1983), 258-265.

[19] G. E. Murphy, The representations of Hecke algebras of type $\mathbf{A}_{\mathbf{n}}$, J. Algebra 173 (1995), 97-121.

[20] G. E. Murphy, Composition factors of the Specht modules for Hecke algebras of type A, J. Alg. 306 (2006), 268-289.

[21] A. Ram, Seminormal representations of Weyl groups and Iwahori-Hecke algebras, Proc. London Math. Soc. (3) 75 (1997), 99-133.

[22] H. Rui and M. Si, Discriminants of Brauer algebras, preprint 2006, math.QA/0607267.

[23] A. Young, On quantitative substitutional analysis II, Proc. Lond. Math. Soc. (3) 34 (1902), 361-397. 


\title{
Appendix. Constructing idempotents from triangular actions
}

\author{
By Marcos Soriano ${ }^{1)}$ at Hannover
}

\begin{abstract}
We give a general construction of a complete set of orthogonal idempotents starting from a set of elements acting in an (upper) triangular fashion. The construction is inspired in the Jucys-Murphy elements (in their various appearances in several cellular algebras).
\end{abstract}

\section{Triangular actions: setup and notation}

The construction of idempotents presented here is based only on matrix arithmetic. However, whenever possible, we will mention the more suggestive notation from combinatorial representation theory.

Let $\Lambda$ be an $R$-algebra, where $R$ is an arbitrary integral domain. The starting point is a representation $\rho$ of $\Lambda$ via matrices over $R$, that is, an $R$-free (left) $\Lambda$-module $M$. Let $d$ be the $R$-rank of $M$ and set $\underline{\boldsymbol{d}}:=\{1, \ldots, d\}$.

1.1. Remark. Until section 5 we will not make any additional assumptions on $R$ or $\Lambda$. We have in mind such examples as $\Lambda$ being a cellular $R$-algebra and $M$ a single cell ("Specht") module $M$, which would give rise to "Young's Orthogonal Form" for $M$, as well as the case $M=\Lambda$ itself, e.g. for questions of semisimplicity.

Assume that with respect to a certain basis (of "tableaux")

$$
\mathscr{T}:=\left\{t_{1}, \ldots, t_{d}\right\} \subset M
$$

there is a finite set of elements $\mathscr{L}:=\left\{L_{1}, \ldots, L_{n}\right\} \subset \Lambda$ (the "Jucys-Murphy" elements) acting in an upper triangular way, that is,

$$
\rho\left(L_{i}\right)=\left(\begin{array}{cccc}
r_{i}^{1} & * & \cdots & * \\
0 & r_{i}^{2} & \ddots & \vdots \\
\vdots & \ddots & \ddots & * \\
0 & \cdots & 0 & r_{i}^{d}
\end{array}\right), \quad \forall i \in \underline{\boldsymbol{n}}
$$

for certain diagonal entries $\left\{r_{i}^{j}\right\}, i \in \underline{\boldsymbol{n}}, j \in \underline{\boldsymbol{d}}$ (the "residues" or "contents"). Call

$$
\left(r_{1}^{j}, r_{2}^{j}, \ldots, r_{n}^{j}\right)
$$

1) The author thanks Andrew Mathas for his generosity and hospitality. 
the residue sequence corresponding to the basis element $t_{j}$. From now on, we identify $L_{i}$ with its representing matrix, thus suppressing $\rho$. Note that we do not make any assumption on $\langle\mathscr{L}\rangle$ being central in $\Lambda$ or that $\mathscr{L}$ consists of pairwise commuting elements.

Finally, we need some notation for matrices. We denote by $\left\{E_{i j}\right\}_{i, j \in \underline{d}}$ the canonical basis of matrix units of $\operatorname{Mat}_{d}(R)$, whose elements multiply according to $E_{i j} E_{k l}=\delta_{j k} E_{i l}$. The subring of $\operatorname{Mat}_{d}(R)$ consisting of upper triangular matrices contains a nilpotent ideal with $R$-basis $\left\{E_{i j}\right\}_{1 \leq i<j \leq d}$ which we denote by $\mathscr{N}$. We define the support of a matrix $A=\left(a_{i j}\right) \in \operatorname{Mat}_{d}(R)$ in the obvious way,

$$
\operatorname{supp}(A):=\left\{(i, j) \in \underline{\boldsymbol{d}} \times \underline{\boldsymbol{d}} \mid a_{i j} \neq 0\right\} .
$$

To any $i \in \underline{\boldsymbol{d}}$ we associate the following subset of $\underline{\boldsymbol{d}}^{2}$ :

$$
\mathfrak{u}_{i}:=\left\{(k, l) \in \underline{\boldsymbol{d}}^{2} \mid k \leqq i \leqq l\right\},
$$

and extend this definition to any non-empty subset $J \subseteq \underline{\boldsymbol{d}}$ via $\mathfrak{u}_{J}:=\bigcup_{i \in J} \mathfrak{u}_{i}$. If $J$ is non-empty then a matrix $A$ has shape $J$ if $\operatorname{supp}(A) \subseteq \mathfrak{u}_{J}$ and the sequence $\left(a_{i i}\right)_{i \in \boldsymbol{d}}$ of diagonal entries is the characteristic function of the subset $J$, that is,

$$
a_{i i}= \begin{cases}1, & \text { if } i \in J, \\ 0, & \text { if } i \notin J .\end{cases}
$$

In particular, $A \in \sum_{i \in J} E_{i i}+\mathscr{N}$ and $A$ is upper triangular. For example, the matrices of shape $\{i\}$ have the form

$$
\left(\begin{array}{ccccccc}
0 & \cdots & 0 & * & * & \cdots & * \\
& \ddots & \vdots & \vdots & & & \vdots \\
& & 0 & * & & & * \\
& & & 1 & * & \cdots & * \\
& & & & 0 & \cdots & 0 \\
& & & & & \ddots & \vdots \\
& & & & & & 0
\end{array}\right) .
$$

\section{Cayley-Hamilton and lifting idempotents}

Let us pause to consider a single upper triangular matrix

$$
Z=\left(\begin{array}{cccc}
\zeta_{1} & * & \cdots & * \\
0 & \zeta_{2} & \ddots & \vdots \\
\vdots & \ddots & \ddots & * \\
0 & \cdots & 0 & \zeta_{d}
\end{array}\right) \in \operatorname{Mat}_{d}(R)
$$


Note that by the Cayley-Hamilton theorem, the matrix $Z$ satisfies the polynomial $\prod_{i=1}^{d}\left(X-\zeta_{i}\right)$. Assume that $Z$ has shape $J$ for some non-empty $J \subseteq \underline{\boldsymbol{d}}$ of cardinality $k=|J|$. Then $Z$ satisfies the polynomial $(X-1)^{k} \cdot X^{d-k}$. What if $k=1$ ? Then the CayleyHamilton equation for $Z$ reads

$$
0=Z^{d-1} \cdot(Z-1) \quad \Leftrightarrow \quad Z^{d}=Z^{d-1} .
$$

This implies (by induction) $Z^{d+j}=Z^{d}$ for all $j \geqq 1$. In particular, the element $F:=Z^{d}$ is an idempotent.

Of course, this is just a special case of "lifting" idempotents, and can be extended (cf. [1], Section I.12) to the following ring theoretical version (Lemma 2.4). We introduce some notation first.

Let $N \geqq 2$ be a natural number (corresponding to the nilpotency degree in Lemma 2.4; for $N=1$ there is nothing to do). Consider the following polynomial in two (commuting) indeterminates:

$$
\begin{aligned}
(X+Y)^{2 N-1} & =\sum_{i=0}^{2 N-1}\left(\begin{array}{c}
2 N-1 \\
i
\end{array}\right) X^{i} Y^{2 N-1-i} \\
& =\sum_{i=0}^{N-1}\left(\begin{array}{c}
2 N-1 \\
i
\end{array}\right) X^{2 N-1-i} Y^{i}+\sum_{i=0}^{N-1}\left(\begin{array}{c}
2 N-1 \\
i
\end{array}\right) X^{i} Y^{2 N-1-i} \\
& =: \varepsilon_{N}(X, Y)+\varepsilon_{N}(Y, X)
\end{aligned}
$$

(using the symmetry of the binomial coefficients). Note that $\varepsilon_{N}(X, Y)$ has integer coefficients. Since $N>1$,

$$
\varepsilon_{N}(X, Y) \equiv X^{2 N-1} \bmod (X Y)
$$

and $\varepsilon_{N}(X, Y) \equiv 0 \bmod \left(X^{N}\right)$. This implies that

$$
\varepsilon_{N}(X, Y) \cdot \varepsilon_{N}(Y, X) \equiv 0 \bmod (X Y)^{N} .
$$

Specialise to a single indeterminate by setting $\varepsilon_{N}(X):=\varepsilon_{N}(X, 1-X)$ and observe that

$$
1=1^{2 N-1}=(X+(1-X))^{2 N-1}=\varepsilon_{N}(X)+\varepsilon_{N}(1-X) .
$$

Now we are ready to formulate the

2.4. Lemma. Let $\mathscr{H}$ be a ring and $\mathscr{N}$ a nilpotent two-sided ideal of nilpotency degree $N$. If $x^{2} \equiv x \bmod \mathscr{N}$, then $e:=\varepsilon_{N}(x)$ is an idempotent with $e \equiv x \bmod \mathscr{N}$.

Proof. Note that $x^{2} \equiv x \bmod \mathscr{N} \Leftrightarrow x-x^{2}=x(1-x) \in \mathscr{N}$, implying

$$
e=\varepsilon_{N}(x) \equiv x^{2 N-1} \equiv x \quad \bmod \mathscr{N}
$$

by equation (2.1). On the other hand, combining equations (2.2) and (2.3), 


$$
e-e^{2}=e(1-e)=\varepsilon_{N}(x) \varepsilon_{N}(1-x) \equiv 0 \quad \bmod (x(1-x))^{N} .
$$

But $(x(1-x))^{N} \in \mathscr{N}^{N}=0$, thus the equality $e-e^{2}=0$ holds and $e$ is indeed an idempotent.

\section{The separating condition and directedness}

We consider first a simple version of the idempotent construction that is relevant to semisimplicity questions. For $i \in \underline{\boldsymbol{d}}$ we denote by $\underline{\hat{\boldsymbol{i}}}$ the set $\underline{\boldsymbol{d}} \backslash\{i\}$. Let us assume now that for all $i \in \underline{\boldsymbol{d}}$ the following separating condition is satisfied:

$$
\forall j \in \underline{\hat{\boldsymbol{i}}} \quad \exists k \in \underline{\boldsymbol{n}} \text { such that } r_{k}^{i}-r_{k}^{j} \in R^{\times} .
$$

In particular, both residues are different. Of course, $k$ may not be unique, but we assume a fixed choice made for all possible pairs of indices. Then we define $(\mathbf{1}$ denotes the identity matrix)

$$
Z_{i}:=\prod_{j \in \underline{\hat{i}}} \frac{L_{k}-r_{k}^{j} \mathbf{1}}{r_{k}^{i}-r_{k}^{j}} .
$$

The product can be taken in any order, the essential fact being only to achieve that the matrix $Z_{i}$ is of the form $Z_{i}=E_{i i}+N_{i}$ for some upper triangular nilpotent matrix $N_{i}$. Just note that for the $j$-th factor $F$ in the definition of $Z_{i}$ we have

$$
F_{i i}=\frac{r_{k}^{i}-r_{k}^{j}}{r_{k}^{i}-r_{k}^{j}}=1 \quad \text { and } \quad F_{j j}=\frac{r_{k}^{j}-r_{k}^{j}}{r_{k}^{i}-r_{k}^{j}}=0 .
$$

Now, using the observation of $\S 2$, we obtain a set of idempotents $\mathscr{E}_{i}:=Z_{i}^{d}$. Our first assertion is

3.1. Lemma. The idempotent $\mathscr{E}_{i}$ has shape $\{i\}$.

Proof. Any matrix of the form $\left(E_{i i}+N\right)^{d}$ with $N \in \mathscr{N}$ has shape $\{i\}$. To see this, use the non-commutative binomial expansion for $U=\left(E_{i i}+N\right)^{d}$, that is, express $U$ as a sum of terms $X_{1} \cdots X_{d}$, where $X_{j} \in\left\{E_{i i}, N\right\}$. If all $X_{j}=N$, we have the (only) summand of the form $N^{d}=0$ (by nilpotency), with no contribution. Similarly, if all $X_{j}=E_{i i}$, we obtain one summand $E_{i i}$.

In the case when $X_{1}$ or $X_{d}$ equals $E_{i i}$, and at least one factor equals $N$, this summand has the appropriate form,

$$
\text { either }\left(\begin{array}{ccccccc}
0 & \cdots & 0 & 0 & 0 & \cdots & 0 \\
& \ddots & \vdots & \vdots & \vdots & & \vdots \\
& & 0 & 0 & 0 & \cdots & 0 \\
& & & 0 & * & \cdots & * \\
& & & & 0 & \cdots & 0 \\
& & & & & \ddots & \vdots \\
& & & & & & 0
\end{array}\right) \text { or }\left(\begin{array}{ccccccc}
0 & \cdots & 0 & * & 0 & \cdots & 0 \\
& \ddots & \vdots & \vdots & \vdots & & \vdots \\
& & 0 & * & 0 & \cdots & 0 \\
& & & 0 & 0 & \cdots & 0 \\
& & & & 0 & \cdots & 0 \\
& & & & & \ddots & \vdots \\
& & & & & & 0
\end{array}\right) \text {. }
$$


Thus we are left with the summands having $X_{1}=X_{d}=N$ and $X_{j}=E_{i i}$ for some $1<j<d$. But the support of any matrix in $\mathscr{N} E_{i i} \mathscr{N}$ is contained in the set $\left\{(k, s) \in \underline{\boldsymbol{d}}^{2} \mid k<i<s\right\}$, as one sees by matrix unit gymnastics (running indices are underlined):

$$
\left(\sum_{\underline{k}<\underline{j}} a_{k j} E_{k j}\right) \cdot E_{i i} \cdot\left(\sum_{\underline{r}<\underline{s}} b_{r s} E_{r s}\right)=\left(\sum_{\underline{k}<\underline{j}} a_{k j} E_{k j}\right)\left(\sum_{i<\underline{s}} b_{i s} E_{i s}\right)=\sum_{1 \leqq \underline{k}<i<\underline{s} \leqq d} a_{k i} b_{i s} E_{k s} .
$$

This finishes the proof of the lemma, as all summands add up to show that $U-E_{i i}$ is nilpotent with support contained in $\mathfrak{u}_{i}$.

Lemma 3.1 has an important consequence: the one-sided "directed" orthogonality of the obtained idempotents.

3.2. Definition. Let $\mathscr{H}$ be an arbitrary ring. Call a finite set $\left\{e_{1}, \ldots, e_{d}\right\}$ of idempotents in $\mathscr{H}$ directed, if $e_{j} e_{i}=0$ whenever $j>i$.

3.3. Lemma. The set of idempotents $\left\{\mathscr{E}_{i}\right\}_{i \in \underline{d}}$ is directed.

Proof. Directedness is an immediate consequence of the fact that $\mathscr{E}_{i}$ has shape $\{i\}$.

\section{Gram-Schmidt orthogonalisation of directed systems of idempotents}

We can now proceed inductively and construct a complete set of orthogonal idempotents out of $\left\{\mathscr{E}_{i}\right\}$. The inductive step goes as follows:

4.1. Lemma. Let $\mathscr{H}$ be an arbitrary ring. Assume we are given two finite sets of idempotents in $\mathscr{H}$ (one of them possibly empty):

$$
\mathbb{E}=\left\{e_{1}, \ldots, e_{k}\right\} \quad \text { and } \quad \mathbb{F}=\left\{f_{k+1}, f_{k+2}, \ldots, f_{d}\right\}
$$

for some $k \geqq 0$ with the following properties:

(a) $\mathbb{E}$ consists of pairwise orthogonal idempotents.

(b) $\mathbb{E}$ is directed.

(c) $\mathbb{E}$ is orthogonal to $\mathbb{F}$, that is, ef $=0=$ fe for e $\in \mathbb{E}, f \in \mathbb{F}$.

Set $F:=\sum_{i=1}^{k} e_{i}+f_{k+1}$. Then the sets of idempotents

$$
\tilde{\mathbb{E}}=\left\{e_{1}, \ldots, e_{k}, f_{k+1}\right\} \quad \text { and } \quad \hat{\mathbb{E}}=\left\{(1-F) f_{k+2}, \ldots,(1-F) f_{d}\right\}
$$

satisfy conditions (a)-(c).

Proof. First observe that $F$ is an idempotent, by orthogonality. If $j \geqq k+2$ we have (by the orthogonality of $\mathbb{E}$ and $\mathbb{F}$ and the directedness of $\mathbb{F}$ ) that 


$$
f_{j} \cdot F=f_{j} \cdot\left(e_{1}+\cdots+e_{k}+f_{k+1}\right)=\sum_{i=1}^{k} f_{j} e_{i}+f_{j} f_{k+1}=0+0=0 .
$$

This implies that $\hat{f}_{j}:=(1-F) f_{j}$ is an idempotent because

$$
\hat{f}_{j}^{2}=\left(f_{j}-F f_{j}\right)\left(f_{j}-F f_{j}\right)=f_{j}-F f_{j}-\underbrace{f_{j} F}_{=0} f_{j}+F \underbrace{f_{j} F}_{=0} f_{j}=(1-F) f_{j}=\hat{f}_{j} .
$$

Similarly, the set $\left\{\hat{f}_{s}\right\}_{k+2 \leqq s \leqq d}$ is directed because for $j>i>k+1$

$$
\hat{f_{j}} \cdot \hat{f_{i}}=\left(f_{j}-F f_{j}\right)\left(f_{i}-F f_{i}\right)=\underbrace{f_{j} f_{i}}_{=0}-\underbrace{f_{j} F}_{=0} f_{i}-F \underbrace{f_{j} f_{i}}_{=0}+F \underbrace{f_{j} F}_{=0} f_{i}=0 .
$$

Since $\mathbb{E}$ is orthogonal to $f_{k+1}$, $\tilde{\mathbb{E}}$ consists obviously of pairwise orthogonal idempotents. Thus, we are left with checking orthogonality between $f_{k+1}$ and $\hat{\mathbb{F}}$. Let $j \geqq k+2$, then

$$
f_{k+1} \cdot \hat{f}_{j}=f_{k+1}(1-F) f_{j}=\underbrace{f_{k+1}\left(1-f_{k+1}\right)}_{=0} f_{j}-\sum_{i=1}^{k} \underbrace{f_{k+1} e_{i} f_{j}}_{=0}=0,
$$

as well as $\hat{f}_{j} \cdot f_{k+1}=(1-F) f_{j} f_{k+1}=0$ by directedness.

Thus, keeping the notations from $\S 1$ and $\S 3$, we obtain the following

4.2. Proposition. A set $\mathscr{L}=\left\{L_{1}, \ldots, L_{n}\right\}$ of "Jucys-Murphy operators" satisfying the separating condition $(\mathscr{S})$ for all $i \in \underline{\boldsymbol{d}}$ gives rise to a complete set of orthogonal idempotents $\left\{e_{1}, \ldots, e_{d}\right\}$.

Proof. Starting from $\mathbb{E}=\emptyset$ and $\mathbb{E}=\left\{\mathscr{E}_{i}=Z_{i}^{d}\right\}_{i \in \underline{d}}$, we obtain-using Lemma $4.1 d$ times - a set $\left\{e_{1}, \ldots, e_{d}\right\}$ of orthogonal idempotents.

Note that the idempotents $e_{i}$ have again shape $\{i\}$ (check this in the inductive step from Lemma 4.1 by considering the form of the matrix $1-F)$. Completeness of the set $\left\{e_{1}, \ldots, e_{d}\right\}$ now follows easily, since we obviously have by Lemma 3.1:

$$
e:=e_{1}+\cdots+e_{d}=\mathbf{1}+N
$$

for some (upper triangular) nilpotent matrix $N$. Thus, $\mathbf{1}-e$ is an idempotent and a nilpotent matrix, implying that $N=0$.

Note that the proof gives, at the same time, a practical algorithm for constructing the complete set of orthogonal idempotents in question.

\section{Linkage classes}

From now on, we assume that $R$ is a local commutative ring with maximal ideal $\mathrm{m}$. This includes the case of $R$ being a field (when $\mathrm{m}=0$ ). 
Fix $k \in \underline{\boldsymbol{n}}$ and $j \in \underline{\boldsymbol{d}}$. We may assume without loss of generality that not all residues $r_{k}^{i}$, $i \in \underline{\boldsymbol{d}}$, are zero (replace $L_{k}$ by $\mathbf{1}+L_{k}$ if necessary ${ }^{2)}$ ). We say that $i \in \underline{\boldsymbol{d}}$ is linked to $j$ via $L_{k}$, if $r_{k}^{i}-r_{k}^{j} \in \mathrm{m}$. Set

$$
\mathbb{Q}_{k}(j):=\left\{i \in \underline{\boldsymbol{d}} \mid i \text { is linked to } j \text { via } L_{k}\right\} .
$$

Observe that $j \in \mathbb{R}_{k}(j)$ since $0 \in \mathfrak{m}$.

5.1. Definition. The linkage class of $j \in \underline{\boldsymbol{d}}$ with respect to $\mathscr{L}=\left\{L_{1}, \ldots, L_{n}\right\}$ is the set

$$
\mathbb{L}(j):=\bigcap_{k \in \underline{n}} \mathbb{L}_{k}(j) .
$$

5.2. Remark. Linkage classes with respect to $\mathscr{L}$ partition the set $\underline{\boldsymbol{d}}$ (of "tableaux") into, say, $l$ disjoint sets $J_{1}, \ldots, J_{l}$. In view of the fact that $R \backslash \mathrm{m}=R^{\times}$, the assumption of the separating condition $(\mathscr{S})$ from $\S 3$ for all $i \in \underline{\boldsymbol{d}}$ just translates into the condition of all linkage classes being singletons.

Consider a fixed linkage class $J$. For all $j \in \underline{\boldsymbol{d}} \backslash J$ we assume that a fixed choice of $k \in \underline{\boldsymbol{n}}$ and $i \in \underline{\boldsymbol{d}}$ has been made such that

$$
r_{k}^{i}-r_{k}^{j} \in R^{\times}=R \backslash \mathrm{m} .
$$

Then we define

$$
Z_{J}:=\prod_{j \notin J} \frac{L_{k}-r_{k}^{j} \mathbf{1}}{r_{k}^{i}-r_{k}^{j}}
$$

(the product can be taken in any order). Note that - by Lemma $3.1-Z_{J}^{d}$ has shape $J$.

\section{A general orthogonalisation algorithm for idempotents}

6.1. Proposition. A set $\mathscr{L}=\left\{L_{1}, \ldots, L_{n}\right\}$ partitioning $\underline{\boldsymbol{d}}$ into l linkage classes gives rise to a complete set $\left\{e_{1}, \ldots, e_{l}\right\}$ of orthogonal idempotents.

Again, the proof of the proposition provides an algorithm for constructing $\left\{e_{1}, \ldots, e_{l}\right\}$.

Proof. Let $J_{1}, \ldots, J_{l}$ denote the linkage classes and set $U_{i}:=Z_{J_{i}}^{d}$, a matrix of shape $J_{i}$. We start the orthogonalisation procedure by setting $\mathbb{E}_{0}:=\emptyset$ and $\mathbb{F}_{l}:=\left\{\varepsilon_{d}\left(U_{i}\right)\right\}_{1 \leqq i \leqq l}$. Note that $\mathbb{F}_{l}$ consists of idempotents by Lemma 2.4. Assuming that two sets of idempotents $\mathbb{E}_{k}=\left\{e_{1}, \ldots, e_{k}\right\}$ (pairwise orthogonal) and $\mathbb{F}_{l-k}=\left\{f_{k+1}, \ldots, f_{l}\right\}$ with $\mathbb{E}_{k}$ orthogonal to $\mathbb{F}_{l-k}$ have been already constructed, we set $\mathbb{E}_{k+1}:=\mathbb{E}_{k} \cup\left\{f_{k+1}\right\}$ and have to modify $\mathbb{F}_{l-k}$ appropriately. The goal is that $\mathbb{F}_{l-k-1}$ consists of idempotents orthogonal to $\mathbb{E}_{k+1}$.

2) Note that this does not change the property of the considered set of Jucys-Murphy operators of being central in $\Lambda$ or, rather, consisting of pairwise commuting elements. 
Set $F:=\sum_{e \in \mathbb{E}_{k+1}} e$ and consider first $\tilde{f}_{j}:=\varepsilon_{d}\left((1-F) f_{j}\right)$ for all $j \geqq k+2$. Since $e(1-F)=0$ for $e \in \mathbb{E}_{k+1}$ and $\varepsilon_{d}(X) \in X^{d} \cdot \mathbb{Z}[X, Y], \mathbb{E}_{k+1}$ is left orthogonal to the idempotent $\tilde{f}_{j}, j \geqq k+2$. Similarly, multiplication from the right by $(1-F)$ and application of the polynomial $\varepsilon_{d}$ forces right orthogonality to hold, while keeping left orthogonality. That is, the set $\mathbb{F}_{l-k-1}=\left\{\hat{f}_{k+2}, \ldots, \hat{f}_{d}\right\}$ with $\hat{f}_{j}:=\varepsilon_{d}\left(\tilde{f}_{j}(1-F)\right)$ has the desired properties.

Thus, after $l$ steps, we end up with an orthogonal set of idempotents $\left\{e_{1}, \ldots, e_{l}\right\}$. Observe that the inductive step described above does not change the shape of the idempotents, implying that $e_{i}$ has shape $J_{i}$, just like the original idempotent $\varepsilon_{d}\left(U_{i}\right)$. This fact, in addition to $J_{1}, \ldots, J_{l}$ partitioning $\underline{\boldsymbol{d}}$, leads to the equation

$$
e_{1}+\cdots+e_{l}=\mathbf{1}-N
$$

with $N$ a nilpotent and idempotent matrix, thus implying $N=0$ and the completeness of $\mathbb{E}_{l}$.

6.2. Remark. Retracing all steps in the proof of Proposition 6.1, we see that the constructed idempotents $e_{i}$ belong to $R\left[L_{1}, \ldots, L_{n}\right]$, the $R$-subalgebra of $\Lambda$ generated by $\mathscr{L}$. Thus, if the elements from $\mathscr{L}$ do commute pairwise, this will still hold for the set of idempotents $\mathbb{E}:=\mathbb{E}_{l}$.

In particular, assuming that $\mathscr{L}$ is a set of central Jucys-Murphy elements for the module $M=\Lambda$, we obtain a set $\mathbb{E}$ of central orthogonal idempotents. Thus, for example, the block decomposition of $\Lambda$ in the case of $R$ being a field must be a refinement of the decomposition into linkage classes induced by $\mathscr{L}$.

We leave the adaptation of the presented methods to particular classes or examples for $\Lambda, R, M$ and $\mathscr{L}$ to the reader's needs.

\section{Reference}

[1] Walter Feit, The Representation Theory of Finite Groups, North-Holland, 1982.

School of Mathematics and Statistics F07, University of Sydney, Sydney NSW 2006, Australia e-mail: a.mathas@usyd.edu.au

Institut für Algebra, Zahlentheorie und Diskrete Mathematik, Leibniz Universität Hannover, Im Welfengarten 1, 30167 Hannover, Deutschland

e-mail: soriano@math.uni-hannover.de

Eingegangen 15. August 2006, in revidierter Fassung 26. Februar 2007 